H. Ackermann, H. Ewe, K.-H. Küfer, M. Schröder Modeling profit sharing in combinatorial exchanges by network flows 
(C) Fraunhofer-Institut für Techno- und Wirtschaftsmathematik ITWM 2011

ISSN 1434-9973

Bericht 205 (2011)

Alle Rechte vorbehalten. Ohne ausdrückliche schriftliche Genehmigung des Herausgebers ist es nicht gestattet, das Buch oder Teile daraus in irgendeiner Form durch Fotokopie, Mikrofilm oder andere Verfahren zu reproduzieren oder in eine für Maschinen, insbesondere Datenverarbeitungsanlagen, verwendbare Sprache zu übertragen. Dasselbe gilt für das Recht der öffentlichen Wiedergabe.

Warennamen werden ohne Gewährleistung der freien Verwendbarkeit benutzt.

Die Veröffentlichungen in der Berichtsreihe des Fraunhofer ITWM können bezogen werden über:

Fraunhofer-Institut für Techno- und Wirtschaftsmathematik ITWM Fraunhofer-Platz 1

67663 Kaiserslautern

Germany

Telefon: $\quad+49(0) 631 / 31600-4674$

Telefax: $\quad+49(0) 631 / 31600-5674$

E-Mail: presse@itwm.fraunhofer.de

Internet: www.itwm.fraunhofer.de 
Das Tätigkeitsfeld des Fraunhofer-Instituts für Techno- und Wirtschaftsmathematik ITWM umfasst anwendungsnahe Grundlagenforschung, angewandte Forschung sowie Beratung und kundenspezifische Lösungen auf allen Gebieten, die für Techno- und Wirtschaftsmathematik bedeutsam sind.

In der Reihe »Berichte des Fraunhofer ITWM « soll die Arbeit des Instituts kontinuierlich einer interessierten Öffentlichkeit in Industrie, Wirtschaft und Wissenschaft vorgestellt werden. Durch die enge Verzahnung mit dem Fachbereich Mathematik der Universität Kaiserslautern sowie durch zahlreiche Kooperationen mit internationalen Institutionen und Hochschulen in den Bereichen Ausbildung und Forschung ist ein großes Potenzial für Forschungsberichte vorhanden. In die Berichtreihe werden sowohl hervorragende Diplom- und Projektarbeiten und Dissertationen als auch Forschungsberichte der Institutsmitarbeiter und Institutsgäste zu aktuellen Fragen der Techno- und Wirtschaftsmathematik aufgenommen.

Darüber hinaus bietet die Reihe ein Forum für die Berichterstattung über die zahlreichen Kooperationsprojekte des Instituts mit Partnern aus Industrie und Wirtschaft.

Berichterstattung heißt hier Dokumentation des Transfers aktueller Ergebnisse aus mathematischer Forschungs- und Entwicklungsarbeit in industrielle Anwendungen und Softwareprodukte - und umgekehrt, denn Probleme der Praxis generieren neue interessante mathematische Fragestellungen.

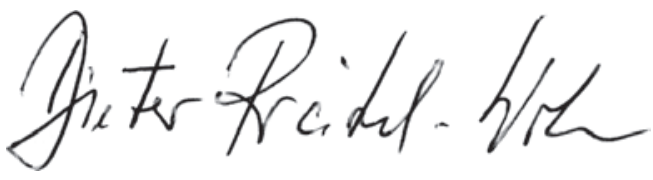

Prof. Dr. Dieter Prätzel-Wolters Institutsleiter

Kaiserslautern, im Juni 2001 



\title{
Modeling Profit Sharing in Combinatorial Exchanges by Network Flows
}

\author{
Heiner Ackermann Hendrik Ewe* Karl-Heinz Küfer \\ Michael Schröder \\ Fraunhofer Institute for Industrial Mathematics \\ Fraunhofer-Platz 1 \\ 67663 Kaiserslautern, Germany ${ }^{\dagger}$
}

April 12, 2011

\begin{abstract}
In this paper we study the possibilities of sharing profit in combinatorial procurement auctions and exchanges. Bundles of heterogeneous items are offered by the sellers, and the buyers can then place bundle bids on sets of these items. That way, both sellers and buyers can express synergies between items and avoid the well-known risk of exposure (see, e.g., [3]). The reassignment of items to participants is known as the Winner Determination Problem (WDP). We propose solving the WDP by using a Set Covering formulation, because profits are potentially higher than with the usual Set Partitioning formulation, and subsidies are unnecessary. The achieved benefit is then to be distributed amongst the participants of the auction, a process which is known as profit sharing.

The literature on profit sharing provides various desirable criteria. We focus on three main properties we would like to guarantee: Budget balance, meaning that no more money is distributed than profit was generated, individual rationality, which guarantees to each player that participation does not lead to a loss, and the core property, which provides every subcoalition with enough money to keep them from separating. We characterize all profit sharing schemes that satisfy these three conditions by a monetary flow network and state necessary conditions on the solution of the WDP for the existence of such a profit sharing. Finally, we establish a connection to the famous VCG payment scheme [2, 8, 19], and the Shapley Value [17].
\end{abstract}

\section{Introduction and Prerequisites}

In this paper we study the possibilities of sharing profit in combinatorial auctions and exchanges. Combinatorial auctions have attracted much attention

\footnotetext{
* Corresponding author, hendrik. ewe@itwm.fraunhofer.de

${ }^{\dagger}$ Part of this work was funded by Stiftung Rheinland-Pfalz für Innovation research grant $961-386261 / 864$.
} 
during the last years, as they promise substantial advantages over classical auctions. While in the latter only a single item is offered on which bidders can place their bids, in combinatorial auctions there are multiple heterogeneous items for sale simultaneously from a single seller. Bidders can use bundle bids to express their valuations, which allows them to avoid the risk of exposure (see, e.g., [3]). Similarly, it can be useful to enable sellers to bundle their items, to let them express their wish to sell only the complete bundle. It is worth mentioning that this is not the same as trading a single "super-item", since the items of the offered bundle may be assigned to different bidders. Combinatorial exchanges take this concept even one step further, as they not only allow multiple buyers, but also multiple sellers, a feature they share with double auctions [18]. Furthermore, they allow participants to simultaneously buy and sell items. Hence, they are a true generalization of combinatorial auctions.

Motivated by our practical problem, a combinatorial freight exchange, we will consider procurement exchanges (also known as reverse exchanges). Here, the items sold are contracts, and buying means delivering the service to the seller. Accordingly, we seek a solution that minimizes procurement costs, whereas in forward exchanges the goal is to maximize the buyers' total valuations. We emphasize again that our model allows bundling both on the seller and the buyer side.

In both combinatorial auctions and exchanges, participants (also known as agents or players) place their offers and bids using a certain bidding language. Although designing such a language can be crucial for the auction's properties, it is also quite domain specific. For a comprehensive overview see [3], for example.

After all offers and bids have been placed, the auctioneer is facing the difficulty of the Winner Determination Problem (WDP). Compared to the singleitem auctions this can be a hard task, as the WDP is equivalent to a Set Packing Problem (SPP) or a Set Covering Problem (SCP), which are both known to be NP-hard in general $[1,5,7]$. We propose to use the SCP formulation, which covers all offered items by at least one bundle, and then resolve the overlapping bundles in a post-processing step. This leads to an unambiguous distribution of items to participants, just as if using the SPP formulation, and can be seen as an automated sub-bundling ${ }^{1}$. One advantage of using SCP rather than SPP is that it increases the solution space and may thereby enhance the auction's revenue. Another nice property is that it does not lead to subsidies ${ }^{2}$, which SPP solutions sometimes do, see Fig. 1. Because of these two reasons, we assume using the SCP formulation with a post-processing step throughout the remaining paper.

Having solved the WDP, the auction's profit has to be distributed among the participants. This process is known as profit sharing, which is the main topic of this paper. The literature on profit sharing provides various interesting properties and criteria, see for example the book by Moulin [11]. We aim to guarantee three properties that are essential for a practical usage of an exchange

\footnotetext{
${ }^{1}$ Note that removing the respective items from the conflicting bundles without penalty is generally no problem in a procurement scenario, as it means executing less service for the same money. One could say it is equivalent to free disposal in a forward auction, and we propose to call it free omission.

${ }^{2}$ In our case, a subsidy occurs if payments are necessary between participants, who did not trade items in the exchange. So, without subsidies all bidding bundles can be paid for by the related offering bundles.
} 




Figure 1: A simple procurement auction with three offering bundles and two bidding bundles. When using the SPP formulation for WDP, subsidies may appear even in optimal solutions. Here, the bidding bundle $(\{A, B\}, 250)$ demands more money for $A$ and $B$ than the offering participants are willing to pay. The optimal solution for the SCP, where items $A, B$, and $C$ fall back to their owners, is also marked for comparison. Here, the two bundles $(\{B, C\}, 100)$ and $(\{C, D\}, 100)$ overlap, which has to be resolved before profit sharing. While the SPP solution generates 750 ,- profit, the SCP model yields $800,-$. In the latter case, to meet the CORE property all profit has to be distributed to the two bundles $(\{C, D\}, 100)$ and $(\{D\}, 900)$, since they can form a subcoalition that generates $800,-$ profit.

platform: budget balance (BB), individual rationality (IR), and the "core property" (CORE). BB means that exactly that much money is distributed as profit was generated, so the exchange does not run at a deficit. IR guarantees to each player that participation does not lead to a personal loss. Besides these two almost inevitable conditions, we propose CORE, which provides every subcoalition with at least as much money as they could have realized among themselves. This keeps them from separating and thereby increases the stability of the profit sharing. It also adds some sense of fairness, since - conversely - not granting the subcoalition the profit they generate would be considered unfair.

With the help of a monetary flow network, we characterize all profit sharing schemes that satisfy these conditions. We show as our main result that every profit sharing scheme that satisfies BB, IR and CORE can be represented by a monetary flow in the network, and vice versa. Furthermore, we establish a connection to the well-known Vickrey-Clarke-Groves (VCG) payment scheme $[2,8,19]$ and the Shapley Value [17]. For some of our results, the solution of the WDP has to satisfy certain criteria. We characterize these conditions and show how they affect the subsequent profit sharing.

We could not find any approach in literature that models profit sharing with the help of a flow network. Many papers explore to which extent a profit sharing scheme can satisfy a set of certain properties simultaneously. One classical result is the Myerson-Satterthwaite theorem [12], which states that no mechanism can be IR, BB and (ex post) efficient, the latter meaning that the total gain from trading items is maximized [3]. Parkes et al. [15, 16] take BB and IR as hard constraints and try to achieve as much efficiency as possible, by lowering par- 
ticipants' incentives to misreport their valuations. The authors give a practical payment rule that minimizes distance to VCG-payments. Day et al. [4] aim at a similar target: Starting from VCG-payments, they implement a mechanism that iteratively generates constraints that force the profit sharing to satisfy $\mathrm{BB}$ and CORE. The constraints are derived from repeatedly solving an NP-hard integer program. Many other papers simply apply the Shapley Value to share profit or costs (see, e.g., [14]). In contrast to these approaches, we do not try to give concrete advice on how to share the profit, but rather outline the framework of possible profit sharing schemes that the monetary flow network allows. By gaining insights from this characterization, one may derive new profit sharing schemes as a future step.

The outline of this paper is as follows. In Section 2 we define our basic notation. In Section 3 we introduce the monetary flow network and show what requirements a solution of the WDP must meet to allow for a profit sharing that is $\mathrm{BB}$, IR, and satisfies CORE. As our main result, we then prove the equivalence of profit sharing schemes with exactly these three properties and monetary flows in the network. In Section 4, we establish the connection between the flow network and the VCG discounts, and show that the Shapley Value can be represented in the flow network. We conclude in Section 5 and give some ideas for further research.

\section{Basic Notation}

In this section we introduce the notation for the remaining paper. Let $A$ be the set of agents (or participants, or players) in the auction and $I$ be the set of items. Agents would like to exchange items amongst each other. They can both offer bundles of items to the other players and also place bundle bids on items offered by other agents. For any offering or bidding bundle $b$, we denote by

$$
\begin{array}{lll} 
& v_{b} \in \mathbb{R}^{\geq 0} & \text { the value of } b, \text { i.e., the offering / bidding price, } \\
\text { and as } & I_{b} \subseteq I & \text { the set of items referred to by } b .
\end{array}
$$

As already mentioned, our context is a procurement auction. Here, for offering players the bundle price $v_{b}$ means the maximum amount of money they are willing to pay for the items in $b$ to be executed. For buyers, on the other hand, it stands for the minimum amount they want to receive if they execute all items in $b$. We call

$$
O:=\left\{b_{1}, \ldots, b_{m}\right\}
$$

the set of offered bundles. As all offered bundles may fall back to their owners, we introduce an artificial bid $\hat{b}_{i}$ for each offered bundle $b_{i} \in O$, with exactly the same properties. We combine all artificial bids and the "real" submitted bids $\left\{b_{m+1}, \ldots, b_{n}\right\}$ into one set

$$
B:=\left\{\hat{b}_{1}, \ldots, \hat{b}_{m}\right\} \cup\left\{b_{m+1}, \ldots, b_{n}\right\}, n>m,
$$

and call it the set of bundle bids. Slightly abusing notation, for a set $\widetilde{B} \subseteq B$, let

$$
I_{\widetilde{B}}:=\bigcup_{b \in \widetilde{B}} I_{b}
$$


be the set of items covered by the bundles in $\widetilde{B}$. A solution $\mathcal{L} \subseteq B$ has the property that $I_{\mathcal{L}}=I$. We call the bundles of a solution $\mathcal{L}$ winning. To simplify further notation, we define for a set $S \subseteq O \cup B$ the set

$$
F_{S}:=\left\{b \in O \mid I_{b} \subseteq I_{S \cap B}\right\}
$$

of satisfiable offers. Note that for any solution $\mathcal{L} \subseteq B$ it holds that $F_{\mathcal{L}}=O$. For a set $S \subseteq(O \cup B)$, we define as

$$
P(S):=\sum_{b \in S \cap F_{S}} v_{b}-\sum_{b \in S \cap B} v_{b}
$$

the profit of $S$. The profit is composed of the value of all offers that can be satisfied by the bundles in $S$ minus the prices of the bidding bundles. Note that $P(\emptyset)=0$. We simply write $P_{\mathcal{L}}$ to refer to a solution's profit $P(O \cup \mathcal{L})=$ $\sum_{b \in O} v_{b}-\sum_{b \in \mathcal{L}} v_{b}$. We call a solution minimal, if $P(S) \leq P_{\mathcal{L}}$ for all subsets $S \subseteq O \cup \mathcal{L}$, and denote it by $\mathcal{L}^{*}$.

\section{Profit Sharing and the Monetary Flow Net- work}

In this section we will prove that every profit sharing scheme that is budget balanced (BB), incentive compatible (IR), and satisfies the core property (CORE) can be described by a monetary flow network, and vice versa. This is a useful result, since all three properties are crucial for practical application and therefore the combination defines a class of highly relevant profit sharing schemes. The flow network gives an intuitive model for sharing profit, and with the help of it we can get a better understanding of the degrees of freedom we have, if we demand BB, IR and CORE. The choices could be used, for example, to fulfill additional criteria focusing on fairness amongst participants, rather than practical operation of an exchange.

Before we proceed with the definition of the monetary flow network, let us formally define the profit sharing function.

Definition 1 (Profit Sharing function). Let $\mathcal{L} \subseteq B$ be a solution. A profit sharing function $p:(O \cup \mathcal{L}) \rightarrow \mathbb{R}$ assigns to each offering or winning bundle $b$ a profit $p(b)$. We sometimes refer to $p$ as a profit sharing scheme interchangeably.

The three essential properties budget balance, individual rationality, and the core property of a profit sharing function $p$ are then defined w.r.t. a solution $\mathcal{L} \subseteq B$ as follows:

$$
\begin{gathered}
\text { BB : } \sum_{b \in O \cup \mathcal{L}} p(b)=P_{\mathcal{L}} \\
\text { IR }: p(b) \geq 0 \forall b \in O \cup \mathcal{L} \\
\text { CORE }: \sum_{b \in S} p(b) \geq P(S) \forall S \subseteq(O \cup \mathcal{L})
\end{gathered}
$$

Please note that we adapted the classical definition of the core property (see, e.g., [13]) slightly to match our scenario: We will always carry out the profit 
sharing on a per bundle basis, since it should not matter in terms of profit share by which participant a certain bundle has been offered. As a nice side effect, this prevents pseudonymous bidding ${ }^{3}$. Nevertheless, all results can be adapted to an agent-based profit sharing. Secondly, we just consider the offering bundles and those bidding bundles belonging to the solution $\mathcal{L}$. One reason is that in combinatorial exchanges with multiple sellers, the CORE constraints can sometimes not be satisfied, if coalitions containing losing bundles are taken into account as well. Because of this, no stable profit sharing can be guaranteed in combinatorial exchanges anyways, and we focus on a stable profit sharing amongst the trading participants. The other reason is that we do not think that a mere participation in the exchange should be rewarded. This would also open the door for fake bidding to obtain some of the profit. Finally, please note again that, by the definition of the achievable profit $P(S)$ in Eq. (1), our definition of CORE builds on the SCP model.

\subsection{Minimal Solutions and Profit Sharing}

It is worth mentioning that the existence of a profit sharing scheme, which satisfies BB, IR, and CORE, depends on the WDP solution it is based on. For non-minimal solutions, there exists a set $S \subseteq \mathcal{L}$ with $P(S)>P_{\mathcal{L}}$. If we now try to distribute profit in a way that satisfies CORE, this set $S$ has to be given more money than the whole solution generates, which in combination with IR directly rules out $\mathrm{BB}$.

A solution is called optimal, if there is no other solution with higher profit. If the WDP is modeled as an SCP, any optimal solution is a minimal solution:

Lemma 1. Any optimal (i.e., profit maximizing) solution $\mathcal{L}$ of the WDP modeled as an $S C P$ is a minimal solution.

Proof. Suppose there was a subset $S \subseteq(O \cup \mathcal{L})$ with $P(S)>P_{\mathcal{L}}$. Then taking just $S$ plus the artificial bids $\hat{b}$ for all offering bundles not completely covered by $S$ is a new solution $\overline{\mathcal{L}}$. The artificial bids generate zero profit, so $P_{\overline{\mathcal{L}}}=P(S)>$ $P_{\mathcal{L}}$, which contradicts the optimality of $\mathcal{L}$.

There might be bundles in $S$ though, which contain items of offering bundles that should now fall back to their owners. More precisely, there are overlaps between the bundles in $S$ and the newly included artificial bids, which have to be resolved. This is only possible in an SCP model.

Please note that Lemma 1 does not necessarily mean that we require an optimal solution. Also non-optimal solutions can be minimal, and there even might be special scenarios, in which every solution found by some solution algorithm is minimal.

\subsection{The Monetary Flow Network}

The distribution of the generated profit amongst the offers and the winning bids of the auction by means of a profit sharing function $p:(O \cup \mathcal{L}) \rightarrow \mathbb{R} \geq 0$ can be easily modeled as a flow network. We introduce two types of nodes: Offering bundles correspond to sources that inject money into the network. On

\footnotetext{
${ }^{3}$ For an overview on pseudonymous bidding in combinatorial auctions see [3].
} 
the other hand, we have sink nodes (corresponding to winning bundles) that are supplied (i.e., paid for) by the network for executing the acquired items. To transfer money between nodes, we have edges from sources to sinks. This naturally results in a bipartite flow network, typical for the so called Hitchcock Problem [9].

An important goal when sharing profit is that the profit should be distributed where it is generated. Accordingly, no payments should be made between players that do not exchange any items, as we do not want subsidies to be necessary. This "localization" property is established by restricting the set of edges to pairs of nodes that trade items:

Definition 2 (Monetary flow network). For a solution $\mathcal{L} \subseteq B$ and the set of offering bundles $O$, define the node-set $O \cup \mathcal{L}$ and the set of edges $E:=\left\{\left(b_{j}, b_{k}\right) \in\right.$ $\left.O \times \mathcal{L} \mid I_{b_{j}} \cap I_{b_{k}} \neq \emptyset\right\}$. We then call $G=(O \cup \mathcal{L}, E)$ a (monetary) flow network for the bundles in $O \cup \mathcal{L}$.

Since we identify nodes with bundles, we usually use the letter $b$ for a node of $G$. Note that the flow network is bipartite, and all $b \in O$ just have outgoing edges while all $b \in \mathcal{L}$ just have incoming edges. A flow is a mapping $f: E \rightarrow \mathbb{R}^{\geq 0}$. For an edge $\left(b_{j}, b_{k}\right) \in E$, we denote by $f\left(b_{j}, b_{k}\right)$ the flow from node $b_{j}$ to node $b_{k}$. In reality, this flow represents a certain amount of money transfered from the owner of $b_{j}$ to the owner of $b_{k}$. We shortly write

$$
f(X, Y):=\sum_{(x, y) \in E \cap(X \times Y)} f(x, y)
$$

for the total flow from the nodes in $X \subseteq O$ to the nodes in $Y \subseteq \mathcal{L}$.

To reflect the freedom of choice in terms of profit sharing, we parametrize the sources' and sinks' capacities in $G$ by a vector $\kappa_{G}:=\left(\kappa_{b}\right)_{b \in O \cup \mathcal{L}}$ : For sources, we set $\kappa_{b} \in\left[0, v_{b}\right]$, while for sinks we have $\kappa_{b} \in\left[-P_{\mathcal{L}},-v_{b}\right]$. So, for an offering bundle, the owner will pay at most his offering price, and for a bidding bundle the owner will get at least the price he asked for. The remaining profit then simply is

$$
p(b)= \begin{cases}v_{b}-\kappa_{b} & \text { for offering bundles } b \in O \\ -\kappa_{b}-v_{b} & \text { for bidding bundles } b \in B\end{cases}
$$

and we can directly deduce the range of $p(b)$ by the ranges for $\kappa_{b}$ :

$$
p(b) \in\left[0, v_{b}\right] \forall b \in O \quad \text { and } \quad p(b) \in\left[0, P_{\mathcal{L}}\right] \forall b \in \mathcal{L} .
$$

Just like for the capacities, we shortly write $p_{G}$ for $(p(b))_{b \in O \times \mathcal{L}}$. Note that we can of course determine the nodes' capacity setup for any given profit distribution $p_{G}$ by solving equation (2) for $\kappa_{b}$. Finally, as in all flow networks, the usual flow conservation constraints should hold:

$$
f(\{b\}, \mathcal{L})-f(O,\{b\})=\kappa_{b} \quad \forall b \in O \cup \mathcal{L}
$$

A flow $f$ in a monetary flow network $G=(O \cup \mathcal{L}, E)$ that obeys constraints (4) with respect to capacities $\kappa_{G}$ is called a valid $\kappa_{G}$-flow. A profit sharing function $p_{G}:(O \cup \mathcal{L}) \rightarrow \mathbb{R}^{\geq 0}$ that can be generated by some valid $\kappa_{G}$-flow in $G$ is called a flow-admitting profit sharing. We define as

$$
\Phi_{G}:=\left\{p_{G} \mid p_{G} \text { is a flow-admitting profit sharing in } G\right\}
$$


the set of all flow-admitting profit sharing schemes for a solution $\mathcal{L}$.

Because we assume that every item is assigned to exactly one bundle (see introduction), we can state the following lemma:

Lemma 2. For all subsets $S \subseteq \mathcal{L}$ of a solution $\mathcal{L} \subseteq B$, in a monetary flow network all edges from nodes in $F_{S}$ lead to nodes in $S$ :

$$
f\left(F_{S}, \mathcal{L} \backslash S\right)=0 .
$$

Proof. Generally, for every bundle $b \in O$ it holds that there is only one edge going out for each item $i \in I_{b}$. By the definition of $F_{S}$, all bundles $b \in F_{S}$ can be completely satisfied by $S$, i.e., there cannot be any $i \in I_{b}$ that is taken by a bid in $\mathcal{L} \backslash S$. Therefore there cannot be any edges and the flow is zero.

\subsection{Properties of the Monetary Flow Network}

In this subsection we will show that the monetary flow network just introduced has some connections to game theoretic properties of profit sharing schemes.

Theorem 1. Let $\mathcal{L} \subseteq B$ be a solution. If $p:(O \cup \mathcal{L}) \rightarrow \mathbb{R}$ is a flow-admitting profit sharing scheme on the corresponding flow network, then $p$ satisfies $\mathrm{BB}$, $\mathrm{IR}$, and CORE.

Proof. First of all, any profit sharing implied by the flow network is of course budget balanced, because of the flow conservation constraints (4) and the bounds on $\kappa_{G}$. As stated in (3) we do not allow negative values for $p$ and therefore we also have individual rationality.

To show that $p$ satisfies CORE, we use some easy set transformations that can be best understood with the help of Figure 3. For all $S \subseteq O \cup \mathcal{L}$ it holds that

$$
\begin{aligned}
\sum_{b \in S} p(b)= & \sum_{b \in S \cap \mathcal{L}} p(b)+\sum_{b \in S \cap O} p(b) \stackrel{(2)}{=} \sum_{b \in S \cap \mathcal{L}}\left(f(O,\{b\})-v_{b}\right)+\sum_{b \in S \cap O}\left(v_{b}-f(\{b\}, \mathcal{L})\right) \\
= & f(O, S \cap \mathcal{L})-f(S \cap O, \mathcal{L})+\left(\sum_{b \in S \cap O} v_{b}-\sum_{b \in S \cap \mathcal{L}} v_{b}\right) \\
= & f(O, S \cap \mathcal{L})-f(S \cap O, \mathcal{L})+\left(\sum_{b \in(S \cap O) \backslash F_{S}} v_{b}+P(S)\right) \\
= & (f(O \backslash S, S \cap \mathcal{L})+f(S \cap O, S \cap \mathcal{L})) \\
& -(f(S \cap O, \mathcal{L} \backslash S)+f(S \cap O, S \cap \mathcal{L}))+\sum_{b \in(S \cap O) \backslash F_{S}} v_{b}+P(S) \\
= & f(O \backslash S, S \cap \mathcal{L})-f(S \cap O, \mathcal{L} \backslash S)+\sum_{b \in(S \cap O) \backslash F_{S}} v_{b}+P(S) \\
= & \underbrace{f(O \backslash S, S \cap \mathcal{L})}_{\geq 0}-\underbrace{f((S \cap O)}_{\leq \sum_{b \in(S \cap O) \backslash F_{S}} v_{b}, \text { since } \kappa_{b} \leq v_{b}} \underbrace{f\left(F_{S} \cap S, \mathcal{L} \backslash S\right)}_{=0 \text { by Lemma } 2}) \\
\geq & P(S) \quad \sum_{b \in(S \cap O) \backslash F_{S}}^{P(S)}+F_{b}(S)
\end{aligned}
$$




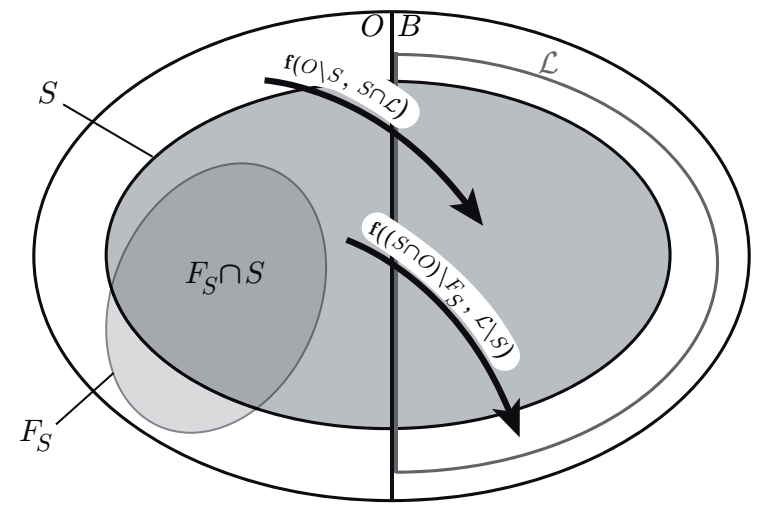

Figure 2: The sets used in Theorem 1. The arrows illustrate two of the terms used in the last transformation of the proof.

Next we will proof the contrary, namely that every profit sharing that obeys $\mathrm{BB}, \mathrm{IR}$, and CORE is a flow-admitting profit sharing scheme:

Theorem 2. Let $\mathcal{L} \subseteq B$ be a solution. If $p$ is a profit sharing w.r.t. $\mathcal{L}$, which satisfies $\mathrm{BB}, \mathrm{IR}$, and $\mathrm{CORE}$, then it is a flow-admitting profit sharing scheme $p \in \Phi_{G}$ on the corresponding monetary flow network $G=(O \cup \mathcal{L}, E)$.

Proof. We set up an extended flow network consisting of a digraph $H=(V, A)$ and arc capacities $u: A \rightarrow \mathbb{R}^{\geq 0}$. The set of nodes consists of all nodes of the monetary flow network $G=(O \cup \mathcal{L}, E)$ and two additional nodes: The source node $s$ is connected to all nodes $v \in O$, and all nodes $v \in \mathcal{L}$ are connected to the sink node $t$. In addition to that, we maintain all edges from $E$. More formally this means that

$$
\begin{aligned}
V & :=\{s, t\} \cup O \cup \mathcal{L} \\
\text { and } \quad A & :=E \cup(\{s\} \times O) \cup(\mathcal{L} \times\{t\}) .
\end{aligned}
$$

The arc capacities are given as follows:

$$
\begin{aligned}
u\left(\left(b_{1}, b_{2}\right)\right) & :=\infty & & \forall\left(b_{1}, b_{2}\right) \in E \\
u((s, b)) & :=v_{b}-p(b) & & \forall(s, b) \in\{s\} \times O \\
u((b, t)) & :=v_{b}+p(b) & & \forall(b, t) \in \mathcal{L} \times\{t\}
\end{aligned}
$$

Note that the arc capacities are chosen in a way that each bundle is able to receive exactly the profit specified by $p$, cf. (2). As $p$ is BB, we have

$$
\begin{aligned}
\sum_{b \in O \cup \mathcal{L}} p(b)=P_{\mathcal{L}} & =\sum_{b \in O} v_{b}-\sum_{b \in \mathcal{L}} v_{b} \\
\Leftrightarrow \sum_{b \in O} v_{b}-\sum_{b \in O} p(b) & =\sum_{b \in \mathcal{L}} v_{b}+\sum_{b \in \mathcal{L}} p(b)
\end{aligned}
$$

and therefore

$$
H^{\max }:=\sum_{b \in O} u((s, b))=\sum_{b \in \mathcal{L}} u((b, t)) .
$$




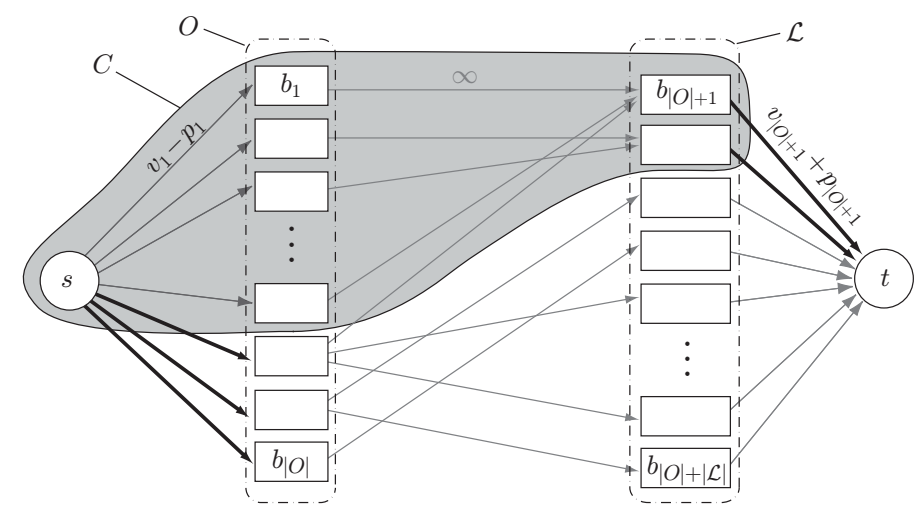

Figure 3: Visualization of a minimum cut as used in the proof of Theorem 2.

We now seek a maximum flow $f^{H}: A \rightarrow \mathbb{R}^{\geq 0}$ in the network $H$. Call

$$
\operatorname{val}\left(f^{H}\right):=\sum_{b \in O} f^{H}(s, b)
$$

the value of the flow. If $\operatorname{val}\left(f^{H}\right)=H^{\text {max }}$, it is easy to see that we have also found a valid flow on the original monetary flow network $G=(O \cup \mathcal{L}, E)$, simply by using the flow values on the edges $O \times \mathcal{L}$.

So assume $\operatorname{val}\left(f^{H}\right)<H^{\max }$. By the MaxFlowMinCuT-Theorem [6], we can then find an $s$-t-cut $C \subseteq V$ whose capacity equals the value of the flow, i.e., a subset of nodes where the leaving edges have a combined capacity of $\operatorname{val}\left(f^{H}\right)$. We assumed that $\operatorname{val}\left(f^{H}\right)<H^{\max }$, so by (6) this cut can neither be $\{s\}$ nor $V \backslash\{t\}$. Furthermore, no edges from $O \times \mathcal{L}$ can leave the cut, since they have infinite capacity. Thus, the edges leaving the cut consist of one or more edges $(s, b), b \in O$, and one or more edges $(b, t), b \in \mathcal{L}$.

We know by (6) that the capacity of $C$ is smaller than the capacity of the cut $C^{\prime}=\{s\}$, and therefore

$$
\sum_{b \in O \backslash C} u((s, b))+\sum_{b \in C \cap \mathcal{L}} u((b, t))<\sum_{b \in O} u((s, b)) .
$$

The corresponding edges are displayed bold in Figure 3. This directly implies

$$
\begin{aligned}
& \sum_{b \in C \cap \mathcal{L}} u((b, t))<\sum_{b \in C \cap O} u((s, b)) \\
\Leftrightarrow & \sum_{b \in C \cap \mathcal{L}} v_{b}+p(b)<\sum_{b \in C \cap O} v_{b}-p(b) \\
\Leftrightarrow & \sum_{b \in C \backslash\{s\}} p(b)<\sum_{b \in C \cap O} v_{b}-\sum_{b \in C \cap \mathcal{L}} v_{b}=P(C \backslash\{s\}),
\end{aligned}
$$

which contradicts the CORE property of $p$. To realize the last equality, please note again that there cannot be any arcs in the cut from $C \cap O$ to $\mathcal{L} \backslash C$, since they have infinite capacity. Therefore $C \cap F_{C}=C \cap O$ and the equality holds (cf. (1)). 
Theorem 1 and Theorem 2 show the equivalence of flows in the monetary flow network and profit sharing schemes that are BB, IR, and CORE. This directly gives us two possibilities to find a profit sharing that satisfies the three properties, or to test a given profit sharing for them: As this type of flow network can be represented by a Linear Program (LP), it can be solved (with any linear objective function, e.g., maximizing a certain bundle's profit) by a polynomial-time algorithm (like the Ellipsoid method or an interior point method). Secondly, one can use specialized algorithms for the Minimum Cost Flow Problem. For an overview see, e.g., the corresponding chapter in [9].

As a final note for this chapter, we would like to emphasize a remarkable implication of Theorem 2: We are able to represent an arbitrary profit sharing that satisfies BB, IR, and CORE as a flow in the monetary flow network for the corresponding solution $\mathcal{L}$. Since the set of edges in this network is restricted to pairs of bundles that exchange items in $\mathcal{L}$, this means that we do not need to transfer money between unrelated bundles, so no subsidies are necessary.

\section{Connection to Existing Approaches}

\subsection{Connection to VCG Discounts}

In this subsection, we use the flow network to share profit amongst the bundles of the exchange. Consider a solution $\mathcal{L} \subseteq B$ and the corresponding monetary flow network $G=(O \cup \mathcal{L}, E)$. For each bundle $b \in O \cup \mathcal{L}$, we define as $p^{*}(b)$ the maximum possible profit that any valid network flow admits:

$$
p^{*}(b):=\max _{p_{G} \in \Phi_{G}} p(b), \quad b \in O \cup \mathcal{L}
$$

For any bundle $b \in O \cup B, p^{*}$ can be imagined as the maximum profit that can be shifted to $b$ through the edges of the network (granting all other bundles at least their required minimum payments). We now show how $p^{*}$ is connected to VCG-discounts that are based on an auction held just amongst the winning bundles.

Theorem 3. Let $\mathcal{L}^{*} \subseteq B$ be a minimal solution and $G=\left(O \cup \mathcal{L}^{*}, E\right)$ the corresponding monetary flow network. Let $p^{*}(b)$ be the maximal profit achievable for some $b \in O \cup \mathcal{L}^{*}$ by any flow-admitting profit sharing $p^{*} \in \Phi_{G}$. Then

$$
p^{*}(b)=P_{\mathcal{L}^{*}}-\Delta_{b},
$$

where

$$
\Delta_{b}:=\max _{S \subseteq O \cup \mathcal{L}^{*} \backslash\{b\}} P(S)
$$

is the maximum profit of a partial exchange of $\mathcal{L}^{*}, b$ is not involved in.

Before we can start the proof, we present some auxiliary definitions and lemmata. The actual proof is then rather short and comprehensive.

Lemma 3 (Supermodularity of $P(\cdot)$ ). For any solution $\mathcal{L} \subseteq B$ and any two subsets $S, T \subset(O \cup \mathcal{L})$, it holds that

$$
P(S \cup T)+P(S \cap T) \geq P(S)+P(T) .
$$


Proof. First, note that

$$
F_{S \cap T}=F_{S} \cap F_{T}
$$

since, on the one hand, $F_{S \cap T} \subseteq F_{S}$ and $F_{S \cap T} \subseteq F_{T}$, and on the other hand it holds that $x \in F_{S} \wedge x \in F_{T} \Rightarrow x \in F_{S \cap T}$. Using this, and realizing by simple counting arguments that

$$
\text { and } \quad \sum_{b \in(S \cup T) \cap\left(F_{S} \cup F_{T}\right)} v_{b}+\sum_{b \in(S \cap T) \cap\left(F_{S} \cap F_{T}\right)}^{F_{S \cup T} \supseteq F_{S} \cup F_{T}} \geq \sum_{b \in S \cap F_{S}} v_{b}+\sum_{b \in T \cap F_{T}} v_{b},
$$

we get:

$$
\begin{aligned}
P & (S \cup T)+P(S \cap T) \\
& =\sum_{b \in(S \cup T) \cap F_{S} \cup T} v_{b}-\sum_{b \in \mathcal{L} \cap(S \cup T)} v_{b}+\sum_{b \in(S \cap T) \cap F_{S \cap T}} v_{b}-\sum_{b \in \mathcal{L} \cap(S \cap T)} v_{b} v_{b}-\sum_{b \in \mathcal{L} \cap(S \cup T)} v_{b}-\sum_{b \in \mathcal{L} \cap(S \cap T)} v_{b} \\
& =\sum_{b \in(S \cap T) \cap\left(F_{S} \cap F_{T}\right)} v_{b}+v_{b} \\
& \underset{(8)}{(8)} \sum_{b \in(S \cup T) \cap\left(F_{S} \cup F_{T}\right)} v_{b}+\sum_{b \in(S \cap T) \cap\left(F_{S} \cap F_{T}\right)} v_{b}-\sum_{b \in \mathcal{L} \cap(S \cup T)} v_{b \in \mathcal{L} \cap(S \cap T)} v_{b}-\sum_{b \in \mathcal{L} \cap S} v_{b}-\sum_{b \in \mathcal{L} \cap T} v_{b} \\
& =\sum_{b \in(S \cup T) \cap\left(F_{S} \cup F_{T}\right)} v_{b}+\sum_{b \in(S \cap T) \cap\left(F_{S} \cap F_{T}\right)} v_{b} v_{b}-\sum_{b \in T \cap F_{T}} v_{b}-\sum_{b \in \mathcal{L} \cap S} v_{b}-\sum_{b \in \mathcal{L} \cap T} \\
& \geq \sum_{(9) S \cap F_{S}} v_{b}+\sum_{b} \\
& =P(S)+P(T)
\end{aligned}
$$

Definition 3. Let $\mathcal{L} \subseteq B$ be a solution. A set $S \subseteq(O \cup \mathcal{L})$ is called tight, if

$$
\forall T \subseteq S: P(T) \leq P(S) .
$$

Note that the concept of tightness may be also used to define a minimal solution: A solution $\mathcal{L}$ is minimal, iff $\mathcal{L} \cup O$ is tight.

Definition 4. Let $\mathcal{L} \subseteq B$ be a solution. The directed graph $\widetilde{G}=(\widetilde{V}, \widetilde{E})$, where

$$
\begin{aligned}
& \widetilde{V}:=\left\{v_{S} \mid S \subseteq O \cup \mathcal{L}, P(S)>0 \text { and } S \text { is tight }\right\} \\
& \text { and } \widetilde{E}:=\left\{\left(v_{S}, v_{T}\right) \mid v_{S}, v_{T} \in \widetilde{V}, S \supset T\right\},
\end{aligned}
$$

is called the partial exchange graph. It contains a node for each subset $S$ of offering and winning bundles that enables a profitable partial exchange of items, and edges from a node to all of its subsets. A leaf node is defined as a node with outdegree zero and represents a minimal partial exchange of $O \cup \mathcal{L}$.

We now present an alternative method to generate a flow-admitting profit sharing (besides the two options mentioned at the end of Section 3).

Algorithm 1. Let $\mathcal{L}^{*}$ be a minimal solution and $G=(O \cup \mathcal{L}, E)$ the corresponding flow network. 
1. Construct $\widetilde{G}=(\widetilde{V}, \widetilde{E})$

2. Set $p(b)=0$ for all $b \in O \cup \mathcal{L}$

3. Repeat until $\widetilde{G}$ has no more nodes:

(a) Pick a leaf node $v_{S} \in \widetilde{V}$

(b) Distribute $\max \left(0, P(S)-\sum_{b \in S} p(b)\right)$ profit arbitrarily amongst the nodes of $S$, adding it to their $p(\cdot)$-values

(c) Remove $v_{S}$ from $\widetilde{V}$

Lemma 4. Algorithm 1 generates a profit distribution $p \in \Phi_{G}$.

Proof. We show that we can apply Theorem 2 to any profit distribution generated by the algorithm. Obviously, CORE and IR are satisfied during the whole algorithm. Untight sets are not a problem, since they receive enough profit by their respective tight subsets. It remains to show that we do not distribute too much profit, i.e., it holds that

$$
\sum_{b \in O \cup \mathcal{L}} p(b) \leq P_{\mathcal{L}}
$$

Let $\left\{T_{1}, \ldots, T_{n}\right\}$ be the sequence of partial exchanges as they are processed by the algorithm. Recall that $T_{j} \subseteq O \cup \mathcal{L}, T_{j}$ is tight, and $P\left(T_{j}\right)>0$ for all $j=1, \ldots, n$. Let

$$
\mathcal{T}_{k}:=\bigcup_{j=1}^{k} T_{j}, \quad \mathcal{T}_{k} \subseteq O \cup \mathcal{L}
$$

be the set of all bids belonging to partial exchanges already processed, including $T_{k}$. Note that $T_{n}=O \cup \mathcal{L}$ and therefore also $\mathcal{T}_{n}=O \cup \mathcal{L}$. Define as $p_{b}^{j}$ the total amount of profit distributed to bundle $b \in(O \cup B)$ directly after processing $v_{T_{j}}$ in the algorithm. We now prove a stronger statement than (10), namely that

$$
\sum_{b \in \mathcal{T}_{j}} p_{b}^{j} \leq P\left(\mathcal{T}_{j}\right) \quad \forall j \in\{1, \ldots, k\},
$$

which contains (10) for $j=n$.

For $j=1$ the statement is obviously true: In the first distribution phase of the algorithm, exactly $P\left(T_{1}\right)$ profit is distributed amongst the nodes of $T_{1}$ and no other profit has been distributed yet. Now assume (11) holds for $j=k$ and the next node to be processed by the algorithm is $v_{T_{k+1}}$. The additional amount of profit that will be distributed is obviously bounded by 0 and $P\left(T_{k+1}\right)$. Besides, if $T_{k+1} \cap \mathcal{T}_{k}$ (see fig. 4) is a partial exchange with strictly positive profit $P\left(T_{k+1} \cap \mathcal{T}_{k}\right)$, the corresponding node $v_{T_{k+1}} \cap \mathcal{T}_{k}$ will already have been processed by the algorithm (as it is a proper subset of $T_{k+1}$ ), so all profit of $v_{T_{k+1} \cap \mathcal{T}_{k}}$ must have been distributed to nodes of $T_{k+1}$. As a direct consequence, the profit newly distributed can be at most $P\left(T_{k+1}\right)-P\left(T_{k+1} \cap \mathcal{T}_{k}\right)$. Recall that by Lemma $3 P(\mathcal{L})$ is supermodular, so it holds that

$$
P\left(T_{k+1} \cup \mathcal{T}_{k}\right)+P\left(T_{k+1} \cap \mathcal{T}_{k}\right) \geq P\left(T_{k+1}\right)+P\left(\mathcal{T}_{k}\right) .
$$




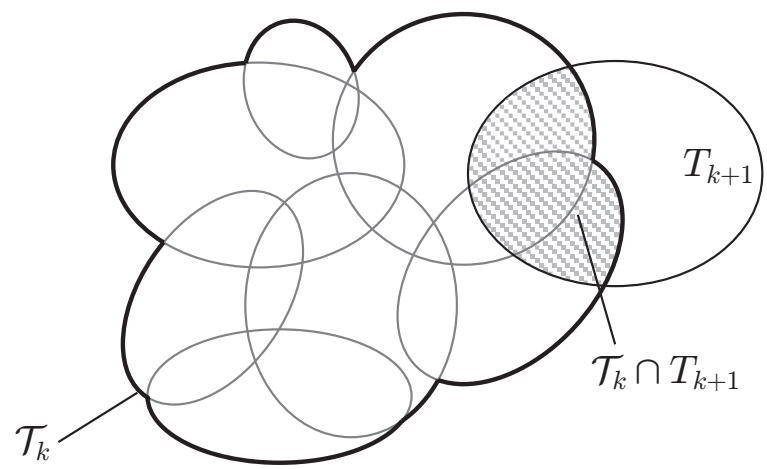

Figure 4: The sets used in the proof of Lemma 4, when processing node $v_{T_{k+1}}$.

Putting everything together, we get the desired statement for $j=k+1$ :

$$
\begin{aligned}
\sum_{b \in \mathcal{T}_{k+1} \cup F_{\mathcal{T}_{k+1}}} p_{b}^{k+1} & \leq \sum_{b \in \mathcal{T}_{k} \cup F_{\mathcal{T}_{k}}} p_{b}^{k}+\left(P\left(T_{k+1}\right)-P\left(T_{k+1} \cap \mathcal{T}_{k}\right)\right) \\
& \leq P\left(\mathcal{T}_{k}\right)+\left(P\left(\mathcal{T}_{k} \cup T_{k+1}\right)-P\left(\mathcal{T}_{k}\right)\right) \\
& =P\left(\mathcal{T}_{k} \cup T_{k+1}\right)=P\left(\mathcal{T}_{k+1}\right)
\end{aligned}
$$

This concludes the proof. The algorithm allows us to directly state that $\Phi_{G} \neq \emptyset$ for an arbitrary minimal solution $\mathcal{L}^{*} \subseteq B$ and the corresponding flow network $G=\left(O \cup \mathcal{L}^{*}, E\right)$ : If we apply the algorithm, we always end up with a flow-admitting profit sharing. Note that the algorithm is related to the algorithm SubmodularCostShare in [13], p.397.

We can now finally prove Theorem 3 with the help of Algorithm 1:

Proof. Let $S^{*}$ be the subset of $\left(O \cup \mathcal{L}^{*}\right) \backslash\{b\}$ with $P\left(S^{*}\right)=\Delta_{b}$. We show that the algorithm can distribute all profit to bundles in $S^{*}$, or to $b$.

Assume the algorithm processes a node belonging to a tight set $T \subseteq(O \cup$ $\left.\mathcal{L}^{*}\right) \backslash\{b\}$. If there exists a substructure $X \subseteq\left(T \backslash S^{*}\right)$ with $P(X)>0$. Then

$$
P\left(S^{*} \cup X\right) \underset{\text { Lemma } 3}{\geq} P\left(S^{*}\right)+P(T)>P\left(S^{*}\right),
$$

which contradicts the maximality of $S^{*}$. Next, note that there cannot be an edge

$$
e \in O \cap\left(S^{*} \backslash T\right) \times \mathcal{L}^{*} \cap\left(T \backslash S^{*}\right),
$$

since this means the bundle $b$, which the edge departs from, cannot be satisfied by $S^{*}$, contradicting its tightness (removing $b$ from $S^{*}$ increases the profit). Similarly, there cannot be an edge $e \in O \cap\left(T \backslash S^{*}\right) \times \mathcal{L}^{*} \cap\left(S^{*} \backslash T\right)$, since that would contradict the tightness of $T$. Hence, all profit of $T$ has to be realized within $S^{*}$. The algorithm will therefore distribute all profit to bundles in $S^{*}$. On the other hand, the profit distributed amongst nodes of $S^{*}$ can only add up to $P\left(S^{*}\right)$ because of eq. (11), so exactly $P\left(S^{*}\right)$ will be distributed. For the remaining sets containing bundle $b$, we can always account the whole profit of 
that node to $b$, which adds up to a total of $P_{\mathcal{L}^{*}}-P\left(S^{*}\right)$. As Algorithm 1 always generates a flow-admitting profit sharing scheme, and as there is no possibility to distribute more than $P_{\mathcal{L}^{*}}-P\left(S^{*}\right)$ to $b$, the statement follows.

Theorem 3 shows an interesting connection. First, recall that the VCGdiscount is calculated as the amount saved by the presence of the particular player, or here: the particular bundle. Applied to just the winning bundles, this gets us exactly $p^{*}(b)$ : It is defined as the solution's original profit minus the profit with $b$ taken out. So it is equivalent to a bundle's VCG-discount in an auction held just amongst the winning bundles.

\subsection{Connection to the Shapley Value}

A profit sharing scheme that is often applied, since it has a couple of nice properties, is the well-known Shapley Value [17]. As we did before, we will apply it to just the offering and the winning bundles and adapt the definition accordingly:

Definition 5 (Shapley Value). Let $\mathcal{L}^{*} \subseteq B$ be a minimal solution. The Shapley Value for bundle $b \in O \cup \mathcal{L}^{*}$ is defined as

$$
p^{\mathrm{sh}}(b)=\sum_{S \subseteq O \cup \mathcal{L}^{*}} \frac{(n-|S|) !(|S|-1) !}{n !}(P(S)-P(S \backslash\{b\})) .
$$

It sort of generalizes the concept of VCG-payments, as it does not only calculate the marginal profit for a bundle when added last to the remaining bundles, but averages over all possible permutations of bundles and all inserting positions. Hence, it does not over-emphasize the threat of a single bundle leaving the coalition, but switches to a more global view, where every bundle's significance is put into perspective of all dependencies.

One can easily show that the Shapley Value is a flow-admitting profit sharing scheme. It is known that for games with a supermodular characteristic function (like $P(\cdot)$, see Lemma 3), the Shapley Value lies in the core, which means it satisfies conditions BB and CORE (see, e.g., [13]). Besides, it is also IR, as $p^{\text {sh }}(b) \geq 0$ for all bundles $b \in O \cup \mathcal{L}^{*}$. Therefore the next corollary directly follows from Theorem 2 .

Corollary 1. Let $\mathcal{L}^{*} \subseteq B$ be a minimal solution and $G=\left(O \cup \mathcal{L}^{*}, E\right)$ the corresponding monetary flow network. Then $p^{\text {sh }}$ is a flow-admitting profit sharing scheme, i.e., $p^{\mathrm{sh}} \in \Phi_{G}$.

So, for all minimal solutions the Shapley Value can be represented by a flow in the corresponding flow network $G=\left(O \cup \mathcal{L}^{*}, E\right)$.

\section{Conclusion and Future Research}

In this paper we examined profit sharing schemes in the context of combinatorial exchanges. We introduced a monetary flow network and were able to show three interesting connections to existing theory. First, every profit sharing scheme that is budget balanced, incentive compatible, and satisfies the core property is equivalent to a flow in a corresponding monetary flow network. This defines 
the class of profit sharing schemes satisfying the three mentioned properties in a very comprehensive way. Secondly, we discovered a connection to the classical VCG discounts. Shifting all profit to a certain bundle within the flow network exactly tells us the VCG discount for that bundle, calculated just within the set of traded bundles. Lastly, we showed that the Shapley Value, applied to the set of traded bundles, can be represented in the flow network.

The question that remains to be answered is, of course, how to use the degrees of freedom that the flow network points out. Easy examples show that simply scaling down $p^{*}$ to force both a relation to VCG payments and keep budget balance may lose the core property, i.e., will result in a profit sharing scheme that is no longer flow-admitting. The same holds for other simple approaches, like an even distribution of the profit to all participants. It remains to show whether there are meaningful and interesting flow-admitting profit sharing schemes besides the Shapley Value. Focusing on fairness amongst participants seems a promising next step.

Finally, it seems worth to further characterize how demanding the concept of a minimal solution is: In which cases is a non-optimal SCP solution still minimal? We are not aware of any literature on such a connection between the employed WDP model, the solution quality, and the properties of the subsequent profit sharing.

\section{Acknowledgments}

We would like to thank Herbert Kopfer from the Chair of Logistics of the University of Bremen. Some of the ideas of this paper were inspired by the paper [10] and from personal communication with him. We thank Ingmar Schüle for his thorough proofreading and fruitful discussions.

\section{References}

[1] E. Balas and M. Padberg, Set partitioning: A survey, SIAM Review, 18 (1976), pp. 710-760.

[2] E. H. Clarke, Multipart pricing of public goods, Public Choice, 18 (1971), pp. 19-33.

[3] P. Cramton, Y. Shoham, and R. Steinberg, Combinatorial Auctions, Mit PR, 2006.

[4] R. W. DAY AND S. RAghavan, Fair payments for efficient allocations in public sector combinatorial auctions, Management Science, 53 (2007), pp. 1389-1406.

[5] S. De VRIES And R. V. Vohra, Combinatorial auctions, INFORMS J. Comput., 15 (2003), pp. 284-309.

[6] L. R. Ford And D. R. Fulkerson, Maximal Flow through a Network., Canadian Journal of Mathematics, 8 (1956), pp. 399-404.

[7] M. R. Garey And D. S. Johnson, Computers and Intractability: A Guide to the Theory of NP-Completeness, W. H. Freeman, January 1979. 
[8] T. Groves, Incentives in teams, Econometrica, 41 (1973), pp. 617-31.

[9] B. Korte And J. Vygen, Combinatorial Optimization: Theory and Algorithms, Springer, Germany, 3rd ed., 2006.

[10] M. A. Krajewska And H. Kopfer, Collaborating freight forwarding enterprises, OR Spectrum, 28 (2006), pp. 301-317.

[11] H. Moulin, Fair division and collective welfare, MIT Press, Cambridge, Mass., 2003.

[12] R. B. Myerson and M. A. Satterthwaite, Efficient mechanisms for bilateral trading, Journal of Economic Theory, 29 (1983), pp. 265-281.

[13] N. Nisan, T. Roughgarden, E. Tardos, and V. V. Vazirani, Algorithmic Game Theory, Cambridge University Press, New York, NY, USA, 2007.

[14] M. Pan, F. Chen, X. Yin, And Y. Fang, Fair profit allocation in the spectrum auction using the Shapley value, in GLOBECOM'09: Proceedings of the 28th IEEE conference on Global telecommunications, Piscataway, NJ, USA, 2009, IEEE Press, pp. 4028-4033.

[15] D. C. Parkes, J. Kalagnanam, and M. Eso, Achieving budget-balance with vickrey-based payment schemes in combinatorial exchanges, tech. report, IBM Research Report RC 22218, 2001. Updated, March 2002.

[16] — Achieving budget-balance with vickrey-based payment schemes in exchanges, in Proceedings of the 17th International Joint Conference on Artificial Intelligence, 2001, pp. 1161-1168.

[17] L. S. Shapley, A value for n-person games, in Contributions to the Theory of Games, Volume II, H. Kuhn and A. Tucker, eds., vol. 28 of Annals of Mathematical Studies, Princeton University Press, 1953, pp. 307-317.

[18] V. L. Smith, An experimental study of competitive market behavior, Journal of Political Economy, 70 (1962), p. 322.

[19] W. Vickrey, Counterspeculation, auctions, and competitive sealed tenders, The Journal of Finance, 16 (1961), pp. 8-37. 


\section{Published reports of the Fraunhofer ITWM}

The PDF-files of the following reports are available under:

\section{www.itwm.fraunhofer.de/de/ zentral_berichte/berichte}

1. D. Hietel, K. Steiner, J. Struckmeier A Finite - Volume Particle Method for Compressible Flows (19 pages, 1998)

2. M. Feldmann, S. Seibold

Damage Diagnosis of Rotors: Application of Hilbert Transform and Multi-Hypothesis Testing

Keywords: Hilbert transform, damage diagnosis,

Kalman filtering, non-linear dynamics

(23 pages, 1998)

3. Y. Ben-Haim, S. Seibold

Robust Reliability of Diagnostic MultiHypothesis Algorithms: Application to Rotating Machinery

Keywords: Robust reliability, convex models, Kalman filtering, multi-hypothesis diagnosis, rotating machinery, crack diagnosis

(24 pages, 1998)

\section{F.-Th. Lentes, N. Siedow}

Three-dimensional Radiative Heat Transfer in Glass Cooling Processes

(23 pages, 1998)

5. A. Klar, R. Wegener

A hierarchy of models for multilane vehicular traffic

Part I: Modeling

(23 pages, 1998)

Part II: Numerical and stochastic investigations (17 pages, 1998)

\section{A. Klar, N. Siedow}

Boundary Layers and Domain Decomposition for Radiative Heat Transfer and Diffusion Equations: Applications to Glass Manufacturing Processes

(24 pages, 1998)

7. I. Choquet

Heterogeneous catalysis modelling and numerical simulation in rarified gas flows Part l: Coverage locally at equilibrium (24 pages, 1998)

8. J. Ohser, B. Steinbach, C. Lang Efficient Texture Analysis of Binary Images (17 pages, 1998)

9. J. Orlik

Homogenization for viscoelasticity of the integral type with aging and shrinkage (20 pages, 1998)

10. J. Mohring

Helmholtz Resonators with Large Aperture (21 pages, 1998)
11. H. W. Hamacher, A. Schöbel On Center Cycles in Grid Graphs (15 pages, 1998)

12. H. W. Hamacher, K.-H. Küfer Inverse radiation therapy planning a multiple objective optimisation approach (14 pages, 1999)

13. C. Lang, J. Ohser, R. Hilfer On the Analysis of Spatial Binary Images (20 pages, 1999)

14. M. Junk

On the Construction of Discrete Equilibrium Distributions for Kinetic Schemes (24 pages, 1999)

15. M. Junk, S. V. Raghurame Rao

A new discrete velocity method for NavierStokes equations

(20 pages, 1999)

16. H. Neunzert

Mathematics as a Key to Key Technologies (39 pages, 1999)

17. J. Ohser, K. Sandau

Considerations about the Estimation of the Size Distribution in Wicksell's Corpuscle Problem

(18 pages, 1999)

18. E. Carrizosa, H. W. Hamacher, R. Klein, S. Nickel

Solving nonconvex planar location problems by finite dominating sets

Keywords: Continuous Location, Polyhedral Gauges, Finite Dominating Sets, Approximation, Sandwich Algorithm, Greedy Algorithm

(19 pages, 2000)

\section{A. Becker}

A Review on Image Distortion Measures Keywords: Distortion measure, human visual system (26 pages, 2000)

20. H. W. Hamacher, M. Labbé, S. Nickel, T. Sonneborn

Polyhedral Properties of the Uncapacitated Multiple Allocation Hub Location Problem Keywords: integer programming, hub location, facility location, valid inequalities, facets, branch and cut (21 pages, 2000)

21. H. W. Hamacher, A. Schöbel

Design of Zone Tariff Systems in Public Transportation

(30 pages, 2001)

22. D. Hietel, M. Junk, R. Keck, D. Teleaga The Finite-Volume-Particle Method for Conservation Laws (16 pages, 2001)

23. T. Bender, H. Hennes, J. Kalcsics, M. T. Melo, S. Nickel

Location Software and Interface with GIS and Supply Chain Management Keywords: facility location, software development, geographical information systems, supply chain management

(48 pages, 2001)
24. H. W. Hamacher, S. A. Tjandra Mathematical Modelling of Evacuation Problems: A State of Art (44 pages, 2001)

25. J. Kuhnert, S. Tiwari

Grid free method for solving the Poisson equation

Keywords: Poisson equation, Least squares method, Grid free method

(19 pages, 2001)

26. T. Götz, H. Rave, D. Reinel-Bitzer, K. Steiner, $\mathrm{H}$. Tiemeier

Simulation of the fiber spinning process Keywords: Melt spinning, fiber model, Lattice Boltzmann, CFD

(19 pages, 2001)

\section{A. Zemitis}

On interaction of a liquid film with an obstacle Keywords: impinging jets, liquid film, models, numerical solution, shape

22 pages, 2001)

\section{I. Ginzburg, K. Steiner}

Free surface lattice-Boltzmann method to model the filling of expanding cavities by Bingham Fluids

Keywords: Generalized LBE, free-surface phenomena interface boundary conditions, filling processes, Bing ham viscoplastic model, regularized models

(22 pages, 2001)

\section{H. Neunzert}

"Denn nichts ist für den Menschen als Menschen etwas wert, was er nicht mit Leidenschaft tun kann

Vortrag anlässlich der Verleihung des Akademiepreises des Landes RheinlandPfalz am 21.11.2001

Keywords: Lehre, Forschung, angewandte Mathematik, Mehrskalenanalyse, Strömungsmechanik (18 pages, 2001)

30. J. Kuhnert, S. Tiwari

Finite pointset method based on the projection method for simulations of the incompressible Navier-Stokes equations

Keywords: Incompressible Navier-Stokes equations, Meshfree method, Projection method, Particle scheme, Least squares approximation

AMS subject classification: 76D05, 76M28

(25 pages, 2001)

31. R. Korn, M. Krekel

Optimal Portfolios with Fixed Consumption or Income Streams

Keywords: Portfolio optimisation, stochastic control, HJB equation, discretisation of control problems (23 pages, 2002)

32. M. Krekel

Optimal portfolios with a loan dependent credit spread

Keywords: Portfolio optimisation, stochastic control, HJB equation, credit spread, log utility, power utility, non-linear wealth dynamics

(25 pages, 2002)

33. J. Ohser, W. Nagel, K. Schladitz

The Euler number of discretized sets - on the choice of adjacency in homogeneous lattices Keywords: image analysis, Euler number, neighborhod relationships, cuboidal lattice

(32 pages, 2002) 
34. I. Ginzburg, K. Steiner

Lattice Boltzmann Model for Free-Surface flow and Its Application to Filling Process in Casting

Keywords: Lattice Boltzmann models; free-surface phenomena; interface boundary conditions; filling processes; injection molding; volume of fluid method; interface boundary conditions; advection-schemes; upwind-schemes

(54 pages, 2002)

35. M. Günther, A. Klar, T. Materne, R. Wegener

Multivalued fundamental diagrams and stop and go waves for continuum traffic equations Keywords: traffic flow, macroscopic equations, kinetic derivation, multivalued fundamental diagram, stop and go waves, phase transitions

(25 pages, 2002)

36. S. Feldmann, P. Lang, D. Prätzel-Wolters Parameter influence on the zeros of network determinants

Keywords: Networks, Equicofactor matrix polynomials, Realization theory, Matrix perturbation theory

(30 pages, 2002)

\section{K. Koch, J. Ohser, K. Schladitz} Spectral theory for random closed sets and estimating the covariance via frequency space

Keywords: Random set, Bartlett spectrum, fast Fourier transform, power spectrum

(28 pages, 2002)

\section{D. d'Humières, I. Ginzburg}

Multi-reflection boundary conditions for lattice Boltzmann models

Keywords: lattice Boltzmann equation, boudary condistions, bounce-back rule, Navier-Stokes equation

(72 pages, 2002)

\section{R. Korn}

\section{Elementare Finanzmathematik}

Keywords: Finanzmathematik, Aktien, Optionen, Portfolio-Optimierung, Börse, Lehrerweiterbildung, Mathematikunterricht

(98 pages, 2002)

40. J. Kallrath, M. C. Müller, S. Nickel

Batch Presorting Problems:

Models and Complexity Results

Keywords: Complexity theory, Integer programming,

Assigment, Logistics

(19 pages, 2002)

\section{J. Linn}

On the frame-invariant description of the phase space of the Folgar-Tucker equation Key words: fiber orientation, Folgar-Tucker equation, injection molding

(5 pages, 2003)

\section{T. Hanne, S. Nickel}

A Multi-Objective Evolutionary Algorithm for Scheduling and Inspection Planning in Software Development Projects

Key words: multiple objective programming, project management and scheduling, software development, evolutionary algorithms, efficient set

(29 pages, 2003)

43. T. Bortfeld, K.-H. Küfer, M. Monz,

A. Scherrer, C. Thieke, H. Trinkaus

Intensity-Modulated Radiotherapy - A Large Scale Multi-Criteria Programming Problem
Keywords: multiple criteria optimization, representative systems of Pareto solutions, adaptive triangulation, clustering and disaggregation techniques, visualization of Pareto solutions, medical physics, external beam radiotherapy planning, intensity modulated radiotherapy (31 pages, 2003)

\section{T. Halfmann, T. Wichmann}

Overview of Symbolic Methods in Industrial Analog Circuit Design

Keywords: $C A D$, automated analog circuit design, sym bolic analysis, computer algebra, behavioral modeling, system simulation, circuit sizing, macro modeling, differential-algebraic equations, index

(17 pages, 2003)

\section{S. E. Mikhailov, J. Orlik}

Asymptotic Homogenisation in Strength and Fatigue Durability Analysis of Composites

Keywords: multiscale structures, asymptotic homogenization, strength, fatigue, singularity, non-local conditions

(14 pages, 2003)

46. P. Domínguez-Marín, P. Hansen, N. Mladenovic, S. Nickel Heuristic Procedures for Solving the Discrete Ordered Median Problem Keywords: genetic algorithms, variable neighborhood search, discrete facility location

(31 pages, 2003)

47. N. Boland, P. Domínguez-Marín, S. Nickel, J. Puerto

Exact Procedures for Solving the Discrete Ordered Median Problem

Keywords: discrete location, Integer programming

(41 pages, 2003)

\section{S. Feldmann, P. Lang}

Padé-like reduction of stable discrete linear systems preserving their stability

Keywords: Discrete linear systems, model reduction,

stability, Hankel matrix, Stein equation

(16 pages, 2003)

49. J. Kallrath, S. Nickel

A Polynomial Case of the Batch Presorting

\section{Problem}

Keywords: batch presorting problem, online optimization, competetive analysis, polynomial algorithms, logistics (17 pages, 2003)

\section{T. Hanne, H. L. Trinkaus}

knowCube for MCDM -

Visual and Interactive Support for

Multicriteria Decision Making

Key words: Multicriteria decision making, knowledge management, decision support systems, visual interfaces, interactive navigation, real-life applications. (26 pages, 2003)

51. O. lliev, V. Laptev

On Numerical Simulation of Flow Through Oil Filters

Keywords: oil filters, coupled flow in plain and porous media, Navier-Stokes, Brinkman, numerical simulation (8 pages, 2003)

52. W. Dörfler, O. Iliev, D. Stoyanov, D. Vassileva On a Multigrid Adaptive Refinement Solver for Saturated Non-Newtonian Flow in Porous Media

Keywords: Nonlinear multigrid, adaptive refinement, non-Newtonian flow in porous media

(17 pages, 2003)
53. S. Kruse

On the Pricing of Forward Starting Options under Stochastic Volatility

Keywords: Option pricing, forward starting options, Heston model, stochastic volatility, cliquet options (11 pages, 2003)

54. O. Iliev, D. Stoyanov

Multigrid - adaptive local refinement solver for incompressible flows

Keywords: Navier-Stokes equations, incompressible flow projection-type splitting, SIMPLE, multigrid methods, adaptive local refinement, lid-driven flow in a cavity (37 pages, 2003)

\section{V. Starikovicius}

The multiphase flow and heat transfer in porous media

Keywords: Two-phase flow in porous media, various formulations, global pressure, multiphase mixture model, numerical simulation

(30 pages, 2003)

\section{P. Lang, A. Sarishvili, A. Wirsen}

Blocked neural networks for knowledge extraction in the software development process Keywords: Blocked Neural Networks, Nonlinear Regression, Knowledge Extraction, Code Inspection (21 pages, 2003)

\section{H. Knaf, P. Lang, S. Zeiser}

Diagnosis aiding in Regulation

Thermography using Fuzzy Logic

Keywords: fuzzy logic, knowledge representation, expert system

(22 pages, 2003)

58. M. T. Melo, S. Nickel, F. Saldanha da Gama Largescale models for dynamic multicommodity capacitated facility location Keywords: supply chain management, strategic planning, dynamic location, modeling (40 pages, 2003)

\section{J. Orlik}

Homogenization for contact problems with periodically rough surfaces

Keywords: asymptotic homogenization, contact problems (28 pages, 2004)

60. A. Scherrer, K.-H. Küfer, M. Monz,

F. Alonso, T. Bortfeld

IMRT planning on adaptive volume structures - a significant advance of computational complexity

Keywords: Intensity-modulated radiation therapy (IMRT), inverse treatment planning, adaptive volume structures, hierarchical clustering, local refinement, adaptive clustering, convex programming, mesh generation, multi-grid methods

(24 pages, 2004)

\section{D. Kehrwald}

Parallel lattice Boltzmann simulation of complex flows

Keywords: Lattice Boltzmann methods, parallel computing, microstructure simulation, virtual material design, pseudo-plastic fluids, liquid composite moulding (12 pages, 2004)

62. O. Iliev, J. Linn, M. Moog, D. Niedziela, V. Starikovicius

On the Performance of Certain Iterative Solvers for Coupled Systems Arising in Discretization of Non-Newtonian Flow Equations 
Keywords: Performance of iterative solvers, Preconditioners, Non-Newtonian flow

(17 pages, 2004)

\section{R. Ciegis, O. Iliev, S. Rief, K. Steiner}

On Modelling and Simulation of Different Regimes for Liquid Polymer Moulding Keywords: Liquid Polymer Moulding, Modelling, Simulation, Infiltration, Front Propagation, non-Newtonian flow in porous media

(43 pages, 2004)

\section{T. Hanne, H. Neu}

Simulating Human Resources in

\section{Software Development Processes}

Keywords: Human resource modeling, software process, productivity, human factors, learning curve

(14 pages, 2004)

\section{O. Iliev, A. Mikelic, P. Popov}

Fluid structure interaction problems in de-

formable porous media: Toward permeability of deformable porous media

Keywords: fluid-structure interaction, deformable porous media, upscaling, linear elasticity, stokes, finite elements

(28 pages, 2004)

66. F. Gaspar, O. Iliev, F. Lisbona, A. Naumovich, P. Vabishchevich

On numerical solution of 1-D poroelasticity equations in a multilayered domain Keywords: poroelasticity, multilayered material, finite volume discretization, MAC type grid

(41 pages, 2004)

67. J. Ohser, K. Schladitz, K. Koch, M. Nöthe Diffraction by image processing and its application in materials science

Keywords: porous microstructure, image analysis, random set, fast Fourier transform, power spectrum, Bartlett spectrum

(13 pages, 2004)

\section{H. Neunzert}

\section{Mathematics as a Technology: Challenges} for the next 10 Years

Keywords: applied mathematics, technology, modelling, simulation, visualization, optimization, glass processing, spinning processes, fiber-fluid interaction, trubulence effects, topological optimization, multicriteria optimiza tion, Uncertainty and Risk, financial mathematics, Malliavin calculus, Monte-Carlo methods, virtual material design, filtration, bio-informatics, system biology

(29 pages, 2004)

69. R. Ewing, O. lliev, R. Lazarov, A. Naumovich On convergence of certain finite difference discretizations for 1D poroelasticity interface problems

Keywords: poroelasticity, multilayered material, finite volume discretizations, MAC type grid, error estimates (26 pages, 2004 )

70. W. Dörfler, O. Iliev, D. Stoyanov, D. Vassileva On Efficient Simulation of Non-Newtonian Flow in Saturated Porous Media with a Multigrid Adaptive Refinement Solver Keywords: Nonlinear multigrid, adaptive renement non-Newtonian in porous media (25 pages, 2004)

\section{J. Kalcsics, S. Nickel, M. Schröde}

Towards a Unified Territory Design Approach - Applications, Algorithms and GIS Integration Keywords: territory desgin, political districting, sales territory alignment, optimization algorithms, Geographical Information Systems

(40 pages, 2005)
72. K. Schladitz, S Peters, D Reinel-Bitzer, A. Wiegmann, J. Ohser

Design of acoustic trim based on geometric modeling and flow simulation for non-woven Keywords: random system of fibers, Poisson line process, flow resistivity, acoustic absorption, Lattice-Boltzmann method, non-woven (21 pages, 2005)

\section{V. Rutka, A. Wiegmann}

Explicit Jump Immersed Interface Method for virtual material design of the effective elastic moduli of composite materials Keywords: virtual material design, explicit jump immersed interface method, effective elastic moduli, composite materials

(22 pages, 2005)

\section{T. Hanne}

Eine Übersicht zum Scheduling von Baustellen Keywords: Projektplanung, Scheduling, Bauplanung, Bauindustrie

(32 pages, 2005)

75. J. Linn

The Folgar-Tucker Model as a Differetial Algebraic System for Fiber Orientation Calculation

Keywords: fiber orientation, Folgar-Tucker model, invariants, algebraic constraints, phase space, trace stability

(15 pages, 2005)

76. M. Speckert, K. Dreßler, H. Mauch, A. Lion, G. J. Wierda

Simulation eines neuartigen Prüfsystems für Achserprobungen durch MKS-Modellierung einschließlich Regelung Keywords: virtual test rig, suspension testing, multibody simulation, modeling hexapod test rig, opti mization of test rig configuration

(20 pages, 2005)

77. K.-H. Küfer, M. Monz, A. Scherrer, P. Süss, F. Alonso, A. S. A. Sultan, Th. Bortfeld,

D. Craft, Chr. Thieke

Multicriteria optimization in intensity modulated radiotherapy planning Keywords: multicriteria optimization, extreme solutions, real-time decision making, adaptive approxima tion schemes, clustering methods, IMRT planning, reverse engineering

(51 pages, 2005)

78. S. Amstutz, H. Andrä

A new algorithm for topology optimization using a level-set method

Keywords: shape optimization, topology optimization topological sensitivity, level-set

(22 pages, 2005)

\section{N. Ettrich}

Generation of surface elevation models for urban drainage simulation

Keywords: Flooding, simulation, urban elevation models, laser scanning

(22 pages, 2005

80. H. Andrä, J. Linn, I. Matei, I. Shklyar, K. Steiner, E. Teichmann

OPTCAST - Entwicklung adäquater Struk turoptimierungsverfahren für Gießereien Technischer Bericht (KURZFASSUNG) Keywords: Topologieoptimierung, Level-Set-Methode Gießprozesssimulation, Gießtechnische Restriktionen, CAE-Kette zur Strukturoptimierung

(77 pages, 2005)
81. N. Marheineke, R. Wegener Fiber Dynamics in Turbulent Flows Part I: General Modeling Framework Keywords: fiber-fluid interaction; Cosserat rod; turbuence modeling: Kolmogorov's energy spectrum; double-velocity correlations; differentiable Gaussian fields (20 pages, 2005)

\section{Part II: Specific Taylor Drag}

Keywords: flexible fibers; $k-\varepsilon$ turbulence model; fiber-turbulence interaction scales; air drag; random Gaussian aerodynamic force; white noise; stochastic differential equations; ARMA process

(18 pages, 2005)

\section{C. H. Lampert, O. Wirjadi}

An Optimal Non-Orthogonal Separation of the Anisotropic Gaussian Convolution Filter Keywords: Anisotropic Gaussian filter, linear filtering, or entation space, $n D$ image processing, separable filters (25 pages, 2005)

83. H. Andrä, D. Stoyanov

Error indicators in the parallel finite element solver for linear elasticity DDFEM Keywords: linear elasticity, finite element method, hierarchical shape functions, domain decom-position, parallel implementation, a posteriori error estimates (21 pages, 2006)

84. M. Schröder, I. Solchenbach Optimization of Transfer Quality in Regional Public Transit

Keywords: public transit, transfer quality, quadratic assignment problem

(16 pages, 2006

85. A. Naumovich, F. J. Gaspar

On a multigrid solver for the three-dimensional Biot poroelasticity system in multilayered domains

Keywords: poroelasticity, interface problem, multigrid, operator-dependent prolongation

(11 pages, 2006)

86. S. Panda, R. Wegener, N. Marheineke Slender Body Theory for the Dynamics of Curved Viscous Fibers

Keywords: curved viscous fibers; fluid dynamics; NavierStokes equations; free boundary value problem; asymptotic expansions; slender body theory

(14 pages, 2006)

87. E. Ivanov, H. Andrä, A. Kudryavtsev Domain Decomposition Approach for Automatic Parallel Generation of Tetrahedral Grids Key words: Grid Generation, Unstructured Grid, Delau nay Triangulation, Parallel Programming, Domain Decomposition, Load Balancing

(18 pages, 2006)

88. S. Tiwari, S. Antonov, D. Hietel, J. Kuhnert, R. Wegener

A Meshfree Method for Simulations of In teractions between Fluids and Flexible Structures

Key words: Meshfree Method, FPM, Fluid Structure Interaction, Sheet of Paper, Dynamical Coupling (16 pages, 2006)

89. R. Ciegis, O. Iliev, V. Starikovicius, K. Steine Numerical Algorithms for Solving Problems of Multiphase Flows in Porous Media Keywords: nonlinear algorithms, finite-volume method software tools, porous media, flows

16 pages, 2006) 
90. D. Niedziela, O. Iliev, A. Latz

On 3D Numerical Simulations of Viscoelastic Fluids

Keywords: non-Newtonian fluids, anisotropic viscosity, integral constitutive equation

(18 pages, 2006)

\section{A. Winterfeld}

Application of general semi-infinite Programming to Lapidary Cutting Problems Keywords: large scale optimization, nonlinear programming, general semi-infinite optimization, design centering, clustering

(26 pages, 2006)

\section{J. Orlik, A. Ostrovska}

Space-Time Finite Element Approximation and Numerical Solution of Hereditary Linear Viscoelasticity Problems

Keywords: hereditary viscoelasticity; kern approximation by interpolation; space-time finite element approximation, stability and a priori estimate

(24 pages, 2006)

93. V. Rutka, A. Wiegmann, H. Andrä EJIIM for Calculation of effective Elastic Moduli in 3D Linear Elasticity

Keywords: Elliptic PDE, linear elasticity, irregular domain, finite differences, fast solvers, effective elastic moduli

(24 pages, 2006)

\section{A. Wiegmann, A. Zemitis}

EJ-HEAT: A Fast Explicit Jump Harmonic Averaging Solver for the Effective Heat Conductivity of Composite Materials Keywords: Stationary heat equation, effective thermal conductivity, explicit jump, discontinuous coefficients, virtual material design, microstructure simulation, EJ-HEAT

(21 pages, 2006)

\section{A. Naumovich}

On a finite volume discretization of the three-dimensional Biot poroelasticity system in multilayered domains

Keywords: Biot poroelasticity system, interface problems, finite volume discretization, finite difference method (21 pages, 2006)

\section{M. Krekel, J. Wenzel}

A unified approach to Credit Default Swaption and Constant Maturity Credit Default Swap valuation

Keywords: LIBOR market model, credit risk, Credit Default Swaption, Constant Maturity Credit Default Swapmethod

(43 pages, 2006)

\section{A. Dreyer}

\section{Interval Methods for Analog Circiuts}

Keywords: interval arithmetic, analog circuits, tolerance analysis, parametric linear systems, frequency response, symbolic analysis, CAD, computer algebra

(36 pages, 2006)

98. N. Weigel, S. Weihe, G. Bitsch, K. Dreßler Usage of Simulation for Design and Optimization of Testing

Keywords: Vehicle test rigs, MBS, control, hydraulics,

testing philosophy

(14 pages, 2006)

99. H. Lang, G. Bitsch, K. Dreßler, M. Speckert Comparison of the solutions of the elastic and elastoplastic boundary value problems
Keywords: Elastic BVP, elastoplastic BVP, variational inequalities, rate-independency, hysteresis, linear kinematic hardening, stop- and play-operator

(21 pages, 2006)

100. M. Speckert, K. Dreßler, H. Mauch MBS Simulation of a hexapod based suspension test rig

Keywords: Test rig, MBS simulation, suspension,

hydraulics, controlling, design optimization

(12 pages, 2006)

101. S. Azizi Sultan, K.-H. Küfer

A dynamic algorithm for beam orientations in multicriteria IMRT planning

Keywords: radiotherapy planning, beam orientation optimization, dynamic approach, evolutionary algorithm, global optimization

(14 pages, 2006)

102. T. Götz, A. Klar, N. Marheineke, R. Wegener A Stochastic Model for the Fiber Lay-down Process in the Nonwoven Production Keywords: fiber dynamics, stochastic Hamiltonian system, stochastic averaging

(17 pages, 2006)

103. Ph. Süss, K.-H. Küfer

Balancing control and simplicity: a variable aggregation method in intensity modulated radiation therapy planning

Keywords: IMRT planning, variable aggregation, clustering methods

(22 pages, 2006)

104. A. Beaudry, G. Laporte, T. Melo, S. Nickel Dynamic transportation of patients in hospitals

Keywords: in-house hospital transportation, dial-a-ride, dynamic mode, tabu search

(37 pages, 2006)

105. Th. Hanne

Applying multiobjective evolutionary algorithms in industrial projects

Keywords: multiobjective evolutionary algorithms, discrete optimization, continuous optimization, electronic circuit design, semi-infinite programming, scheduling (18 pages, 2006)

106. J. Franke, S. Halim

Wild bootstrap tests for comparing signals and images

Keywords: wild bootstrap test, texture classification, textile quality control, defect detection, kernel estimate, nonparametric regression

(13 pages, 2007)

107. Z. Drezner, S. Nickel

Solving the ordered one-median problem in the plane

Keywords: planar location, global optimization, ordered median, big triangle small triangle method, bounds, numerical experiments

(21 pages, 2007)

108. Th. Götz, A. Klar, A. Unterreiter, R. Wegener

Numerical evidance for the non-existing of solutions of the equations desribing rotational fiber spinning

Keywords: rotational fiber spinning, viscous fibers, boundary value problem, existence of solutions (11 pages, 2007)
109. Ph. Süss, K.-H. Küfer

Smooth intensity maps and the BortfeldBoyer sequencer

Keywords: probabilistic analysis, intensity modulated radiotherapy treatment (IMRT), IMRT plan application step-and-shoot sequencing

(8 pages, 2007)

110. E. Ivanov, O. Gluchshenko, H. Andrä, A. Kudryavtsev

Parallel software tool for decomposing and meshing of $3 d$ structures

Keywords: a-priori domain decomposition, unstructured grid, Delaunay mesh generation

(14 pages, 2007)

111. O. lliev, R. Lazarov, J. Willems Numerical study of two-grid preconditioners for 1d elliptic problems with highly oscillating discontinuous coefficients Keywords: two-grid algorithm, oscillating coefficients, preconditioner

(20 pages, 2007)

112. L. Bonilla, T. Götz, A. Klar, N. Marheineke, R. Wegener

Hydrodynamic limit of the Fokker-Planckequation describing fiber lay-down processes

Keywords: stochastic dierential equations, FokkerPlanck equation, asymptotic expansion, Ornstein-

Uhlenbeck process

(17 pages, 2007)

\section{S. Rief}

Modeling and simulation of the pressing section of a paper machine

Keywords: paper machine, computational fluid dynamics, porous media

(41 pages, 2007)

114. R. Ciegis, O. Iliev, Z. Lakdawala

On parallel numerical algorithms for simulating industrial filtration problems

Keywords: Navier-Stokes-Brinkmann equations, finite volume discretization method, SIMPLE, parallel computing, data decomposition method

(24 pages, 2007)

\section{N. Marheineke, R. Wegener}

Dynamics of curved viscous fibers with surface tension

Keywords: Slender body theory, curved viscous bers with surface tension, free boundary value problem (25 pages, 2007)

116. S. Feth, J. Franke, M. Speckert Resampling-Methoden zur mse-Korrektur und Anwendungen in der Betriebsfestigkeit Keywords: Weibull, Bootstrap, Maximum-Likelihood, Betriebsfestigkeit

(16 pages, 2007)

117. H. Knaf

Kernel Fisher discriminant functions - a concise and rigorous introduction

Keywords: wild bootstrap test, texture classification, textile quality control, defect detection, kernel estimate, nonparametric regression

(30 pages, 2007)

118. O. Iliev, I. Rybak

On numerical upscaling for flows in heterogeneous porous media 
Keywords: numerical upscaling heterogeneous porous media, single phase flow, Darcy's law, multiscale problem, effective permeability, multipoint flux approximation, anisotropy

(17 pages, 2007)

\section{O. Iliev, I. Rybak}

On approximation property of multipoint flux approximation method

Keywords: Multipoint flux approximation, finite volume method, elliptic equation, discontinuous tensor coeffi-

cients, anisotropy

(15 pages, 2007)

120. O. Iliev, I. Rybak, J. Willems

On upscaling heat conductivity for a class of industrial problems

Keywords: Multiscale problems, effective heat conductivity, numerical upscaling, domain decomposition

(21 pages, 2007)

121. R. Ewing, O. Iliev, R. Lazarov, I. Rybak On two-level preconditioners for flow in porous media

Keywords: Multiscale problem, Darcy's law, single phase flow, anisotropic heterogeneous porous media, numerical upscaling, multigrid, domain decomposition, efficient preconditioner

(18 pages, 2007)

122. M. Brickenstein, A. Dreyer

POLYBORI: A Gröbner basis framework for Boolean polynomials

Keywords: Gröbner basis, formal verification, Boolean polynomials, algebraic cryptoanalysis, satisfiability

(23 pages, 2007)

\section{O. Wirjad}

Survey of $3 d$ image segmentation methods Keywords: image processing, 3d, image segmentation, binarization

(20 pages, 2007)

\section{S. Zeytun, A. Gupta}

A Comparative Study of the Vasicek and the CIR Model of the Short Rate

Keywords: interest rates, Vasicek model, CIR-model,

calibration, parameter estimation

(17 pages, 2007)

\section{G. Hanselmann, A. Sarishvili}

Heterogeneous redundancy in software quality prediction using a hybrid Bayesian approach

Keywords: reliability prediction, fault prediction, nonhomogeneous poisson process, Bayesian model averaging

(17 pages, 2007)

126. V. Maag, M. Berger, A. Winterfeld, K.-H Küfer

A novel non-linear approach to minimal area rectangular packing

Keywords: rectangular packing, non-overlapping constraints, non-linear optimization, regularization, relaxation

(18 pages, 2007)

127. M. Monz, K.-H. Küfer, T. Bortfeld, C. Thieke Pareto navigation - systematic multi-criteria-based IMRT treatment plan determination

Keywords: convex, interactive multi-objective optimization, intensity modulated radiotherapy planning

(15 pages, 2007)
128. M. Krause A Scherrer

On the role of modeling parameters in IMRT plan optimization

Keywords: intensity-modulated radiotherapy (IMRT) inverse IMRT planning, convex optimization, sensitivity analysis, elasticity, modeling parameters, equivalent uniform dose (EUD)

(18 pages, 2007)

\section{A. Wiegmann}

Computation of the permeability of porous materials from their microstructure by FFFStokes

Keywords: permeability, numerical homogenization

fast Stokes solver

(24 pages, 2007)

130. T. Melo, S. Nickel, F. Saldanha da Gama Facility Location and Supply Chain Management - A comprehensive review

Keywords: facility location, supply chain management network design

(54 pages, 2007)

131. T. Hanne, T. Melo, S. Nickel

Bringing robustness to patient flow management through optimized patient transports in hospitals

Keywords: Dial-a-Ride problem, online problem, case study, tabu search, hospital logistics

(23 pages, 2007)

132. R. Ewing, O. Iliev, R. Lazarov, I. Rybak, J. Willems

An efficient approach for upscaling properties of composite materials with high contrast of coefficients

Keywords: effective heat conductivity, permeability of fractured porous media, numerical upscaling, fibrous insulation materials, metal foams

(16 pages, 2008)

133. S. Gelareh, S. Nickel

New approaches to hub location problems in public transport planning Keywords: integer programming, hub location, transportation, decomposition, heuristic

(25 pages, 2008)

134. G. Thömmes, J. Becker, M. Junk, A. K. Vaikuntam, D. Kehrwald, A. Klar, K. Steiner, A. Wiegmann

A Lattice Boltzmann Method for immiscible multiphase flow simulations using the Level Set Method

Keywords: Lattice Boltzmann method, Level Set method, free surface, multiphase flow (28 pages, 2008

\section{J. Orlik}

Homogenization in elasto-plasticity

Keywords: multiscale structures, asymptotic homogenization, nonlinear energy

(40 pages, 2008)

136. J. Almquist, H. Schmidt, P. Lang, J. Deitmer, M. Jirstrand, D. Prätzel-Wolters, H. Becker

Determination of interaction between MCT1 and CAII via a mathematical and physiological approach

Keywords: mathematical modeling; model reduction: electrophysiology; $\mathrm{pH}$-sensitive microelectrodes; proton antenna

(20 pages, 2008)
137. E. Savenkov, H. Andrä, O. Iliev

An analysis of one regularization approach for solution of pure Neumann problem Keywords: pure Neumann problem, elasticity, regularization, finite element method, condition number (27 pages, 2008)

138. O. Berman, J. Kalcsics, D. Krass, S. Nickel The ordered gradual covering location problem on a network

Keywords: gradual covering, ordered median function, network location

(32 pages, 2008)

139. S. Gelareh, S. Nickel

Multi-period public transport design: A novel model and solution approaches Keywords: Integer programming, hub location, public transport, multi-period planning, heuristics (31 pages, 2008)

140. T. Melo, S. Nickel, F. Saldanha-da-Gama Network design decisions in supply chain planning

Keywords: supply chain design, integer programming models, location models, heuristics

(20 pages, 2008)

141. C. Lautensack, A. Särkkä, J. Freitag K. Schladitz

Anisotropy analysis of pressed point processes

Keywords: estimation of compression, isotropy test nearest neighbour distance, orientation analysis, polar ice, Ripley's K function

(35 pages, 2008)

142. O. Iliev, R. Lazarov, J. Willems

A Graph-Laplacian approach for calculating the effective thermal conductivity of complicated fiber geometries

Keywords: graph laplacian, effective heat conductivity, numerical upscaling, fibrous materials

(14 pages, 2008)

143. J. Linn, T. Stephan, J. Carlsson, R. Bohlin Fast simulation of quasistatic rod deformations for VR applications

Keywords: quasistatic deformations, geometrically exact rod models, variational formulation, energy minimization, finite differences, nonlinear conjugate gradients

(7 pages, 2008)

\section{J. Linn, T. Stephan}

Simulation of quasistatic deformations using discrete rod models

Keywords: quasistatic deformations, geometrically exact rod models, variational formulation, energy minimization, finite differences, nonlinear conjugate gradients

(9 pages, 2008

145. J. Marburger, N. Marheineke, R. Pinnau Adjoint based optimal control using meshless discretizations

Keywords: Mesh-less methods, particle methods, Eulerian-Lagrangian formulation, optimization strategies, adjoint method, hyperbolic equations

(14 pages, 2008

\section{S. Desmettre, J. Gould, A. Szimayer}

Own-company stockholding and work effort preferences of an unconstrained executive Keywords: optimal portfolio choice, executive compensation

(33 pages, 2008) 
147. M. Berger, M. Schröder, K.-H. Küfer A constraint programming approach for the two-dimensional rectangular packing problem with orthogonal orientations

Keywords: rectangular packing, orthogonal orientations non-overlapping constraints, constraint propagation

(13 pages, 2008)

148. K. Schladitz, C. Redenbach, T. Sych, M. Godehardt

Microstructural characterisation of open foams using $3 d$ images

Keywords: virtual material design, image analysis, open foams

(30 pages, 2008)

149. E. Fernández, J. Kalcsics, S. Nickel, R. Ríos-Mercado

A novel territory design model arising in the implementation of the WEEE-Directive Keywords: heuristics, optimization, logistics, recycling (28 pages, 2008)

150. H. Lang, J. Linn

Lagrangian field theory in space-time for geometrically exact Cosserat rods Keywords: Cosserat rods, geometrically exact rods, small strain, large deformation, deformable bodies, Lagrangian field theory, variational calculus (19 pages, 2009)

151. K. Dreßler, M. Speckert, R. Müller, Ch. Weber

Customer loads correlation in truck engineering

Keywords: Customer distribution, safety critical components, quantile estimation, Monte-Carlo methods (11 pages, 2009)

152. H. Lang, K. Dreßler

An improved multiaxial stress-strain correction model for elastic FE postprocessing Keywords: Jiang's model of elastoplasticity, stress-strain correction, parameter identification, automatic differentiation, least-squares optimization, Coleman-Li algorithm

(6 pages, 2009)

153. J. Kalcsics, S. Nickel, M. Schröder A generic geometric approach to territory design and districting

Keywords: Territory design, districting, combinatorial optimization, heuristics, computational geometry (32 pages, 2009)

154. Th. Fütterer, A. Klar, R. Wegener An energy conserving numerical scheme for the dynamics of hyperelastic rods

Keywords: Cosserat rod, hyperealstic, energy conservation, finite differences

(16 pages, 2009)

155. A. Wiegmann, L. Cheng, E. Glatt, O. Iliev, S. Rief

Design of pleated filters by computer simulations

Keywords: Solid-gas separation, solid-liquid separation, pleated filter, design, simulation

(21 pages, 2009)

156. A. Klar, N. Marheineke, R. Wegener Hierarchy of mathematical models for production processes of technical textiles
Keywords: Fiber-fluid interaction, slender-body theory, turbulence modeling, model reduction, stochastic differential equations, Fokker-Planck equation, asymptotic expansions, parameter identification

(21 pages, 2009)

157. E. Glatt, S. Rief, A. Wiegmann, M. Knefel, E. Wegenke

Structure and pressure drop of real and virtual metal wire meshes

Keywords: metal wire mesh, structure simulation, model calibration, CFD simulation, pressure loss (7 pages, 2009)

\section{S. Kruse, M. Mülle}

Pricing American call options under the assumption of stochastic dividends - An application of the Korn-Rogers model Keywords: option pricing, American options, dividends, dividend discount model, Black-Scholes model (22 pages, 2009)

159. H. Lang, J. Linn, M. Arnold

Multibody dynamics simulation of geometrically exact Cosserat rods

Keywords: flexible multibody dynamics, large deformations, finite rotations, constrained mechanical systems, structural dynamics

(20 pages, 2009)

160. P. Jung, S. Leyendecker, J. Linn, M. Ortiz Discrete Lagrangian mechanics and geometrically exact Cosserat rods

Keywords: special Cosserat rods, Lagrangian mechanics, Noether's theorem, discrete mechanics, frame-indifference, holonomic constraints

(14 pages, 2009)

161. M. Burger, K. Dreßler, A. Marquardt, M. Speckert

Calculating invariant loads for system simulation in vehicle engineering

Keywords: iterative learning control, optimal control

theory, differential algebraic equations (DAEs)

(18 pages, 2009)

162. M. Speckert, N. Ruf, K. Dreßler Undesired drift of multibody models excited by measured accelerations or forces Keywords: multibody simulation, full vehicle model, force-based simulation, drift due to noise (19 pages, 2009)

163. A. Streit, K. Dreßler, M. Speckert, J. Lichter, T. Zenner, P. Bach

Anwendung statistischer Methoden zur Erstellung von Nutzungsprofilen für die Auslegung von Mobilbaggern

Keywords: Nutzungsvielfalt, Kundenbeanspruchung, Bemessungsgrundlagen

(13 pages, 2009)

164. I. Correia, S. Nickel, F. Saldanha-da-Gama The capacitated single-allocation hub location problem revisited: A note on a classical formulation

Keywords: Capacitated Hub Location, MIP formulations (10 pages, 2009)

165. F. Yaneva, T. Grebe, A. Scherrer

An alternative view on global radiotherapy optimization problems

Keywords: radiotherapy planning, path-connected sublevelsets, modified gradient projection method, improving and feasible directions

(14 pages, 2009)
166. J. I. Serna, M. Monz, K.-H. Küfer, C. Thieke Trade-off bounds and their effect in multicriteria IMRT planning

Keywords: trade-off bounds, multi-criteria optimization, IMRT, Pareto surface

(15 pages, 2009)

167. W. Arne, N. Marheineke, A. Meister, R. Wegener

Numerical analysis of Cosserat rod and string models for viscous jets in rotational spinning processes

Keywords: Rotational spinning process, curved viscous fibers, asymptotic Cosserat models, boundary value problem, existence of numerical solutions

(18 pages, 2009)

168. T. Melo, S. Nickel, F. Saldanha-da-Gama An LP-rounding heuristic to solve a multiperiod facility relocation problem

Keywords: supply chain design, heuristic, linear programming, rounding

(37 pages, 2009)

169. I. Correia, S. Nickel, F. Saldanha-da-Gama Single-allocation hub location problems with capacity choices

Keywords: hub location, capacity decisions, MILP formulations

(27 pages, 2009)

170. S. Acar, K. Natcheva-Acar

A guide on the implementation of the

Heath-Jarrow-Morton Two-Factor Gaussian

Short Rate Model (HJM-G2++)

Keywords: short rate model, two factor Gaussian,

G2++, option pricing, calibration

(30 pages, 2009)

171. A. Szimayer, G. Dimitroff, S. Lorenz A parsimonious multi-asset Heston model: calibration and derivative pricing

Keywords: Heston model, multi-asset, option pricing, calibration, correlation

(28 pages, 2009)

\section{N. Marheineke, R. Wegener}

Modeling and validation of a stochastic drag for fibers in turbulent flows

Keywords: fiber-fluid interactions, long slender fibers, turbulence modelling, aerodynamic drag, dimensional analysis, data interpolation, stochastic partial differential algebraic equation, numerical simulations, experimental validations

(19 pages, 2009)

173. S. Nickel, M. Schröder, J. Steeg Planning for home health care services Keywords: home health care, route planning, metaheuristics, constraint programming

(23 pages, 2009)

174. G. Dimitroff, A. Szimayer, A. Wagner Quanto option pricing in the parsimonious Heston model

Keywords: Heston model, multi asset, quanto options, option pricing

(14 pages, 2009) 174. G. Dimitroff, A. Szimayer, A. Wagner

175. S. Herkt, K. Dreßler, R. Pinnau

Model reduction of nonlinear problems in structural mechanics

Keywords: flexible bodies, FEM, nonlinear model reduction, POD

(13 pages, 2009) 
176. M. K. Ahmad, S. Didas, J. Iqbal

Using the Sharp Operator for edge detection and nonlinear diffusion

Keywords: maximal function, sharp function, image processing, edge detection, nonlinear diffusion

(17 pages, 2009)

177. M. Speckert, N. Ruf, K. Dreßler, R. Müller, C. Weber, S. Weihe

Ein neuer Ansatz zur Ermittlung von Erprobungslasten für sicherheitsrelevante Bauteile

Keywords: sicherheitsrelevante Bauteile, Kundenbeanspruchung, Festigkeitsverteilung, Ausfallwahrscheinlichkeit, Konfidenz, statistische Unsicherheit, Sicher-

heitsfaktoren

(16 pages, 2009)

\section{J. Jegorovs}

Wave based method: new applicability areas Keywords: Elliptic boundary value problems, inhomogeneous Helmholtz type differential equations in bounded domains, numerical methods, wave based method, uniform B-splines

(10 pages, 2009)

179. H. Lang, M. Arnold

Numerical aspects in the dynamic simulation of geometrically exact rods Keywords: Kirchhoff and Cosserat rods, geometrically exact rods, deformable bodies, multibody dynamics, artial differential algebraic equations, method of lines, time integration

(21 pages, 2009)

180. H. Lang

Comparison of quaternionic and rotationfree null space formalisms for multibody dynamics

Keywords: Parametrisation of rotations, differentialalgebraic equations, multibody dynamics, constrained mechanical systems, Lagrangian mechanics

(40 pages, 2010)

181. S. Nickel, F. Saldanha-da-Gama, H.-P. Ziegler Stochastic programming approaches for risk aware supply chain network design problems Keywords: Supply Chain Management, multi-stage stochastic programming, financial decisions, risk (37 pages, 2010)

\section{P. Ruckdeschel, N. Horbenko}

Robustness properties of estimators in generalized Pareto Models

Keywords: global robustness, local robustness, finite sample breakdown point, generalized Pareto distribution (58 pages, 2010)

\section{P. Jung, S. Leyendecker, J. Linn, M. Ortiz}

A discrete mechanics approach to Cosserat rod theory - Part 1: static equilibria

Keywords: Special Cosserat rods; Lagrangian mechanics; Noether's theorem; discrete mechanics; frameindifference; holonomic constraints; variational formulation

(35 pages, 2010)

184. R. Eymard, G. Printsypar

A proof of convergence of a finite volume scheme for modified steady Richards' equation describing transport processes in the pressing section of a paper machine Keywords: flow in porous media, steady Richards' equation, finite volume methods, convergence of approximate solution

(14 pages, 2010)

185. P. Ruckdeschel

\section{Optimally Robust Kalman Filtering}

Keywords: robustness, Kalman Filter, innovation outlier, additive outlier

(42 pages, 2010)

186. S. Repke, N. Marheineke, R. Pinnau On adjoint-based optimization of a free surface Stokes flow

Keywords: film casting process, thin films, free surface Stokes flow, optimal control, Lagrange formalism (13 pages, 2010)

187. O. Iliev, R. Lazarov, J. Willems

Variational multiscale Finite Element Method for flows in highly porous media Keywords: numerical upscaling, flow in heterogeneous porous media, Brinkman equations, Darcy's law, subgrid approximation, discontinuous Galerkin mixed FEM (21 pages, 2010)

\section{S. Desmettre, A. Szimayer}

Work effort, consumption, and portfolio selection: When the occupational choice matters

Keywords: portfolio choice, work effort, consumption, occupational choice

(34 pages, 2010)

189. O. Iliev, Z. Lakdawala, V. Starikovicius On a numerical subgrid upscaling algorithm for Stokes-Brinkman equations

Keywords: Stokes-Brinkman equations, subgrid approach, multiscale problems, numerical upscaling (27 pages, 2010)

190. A. Latz, J. Zausch, O. Iliev

Modeling of species and charge transport in Li-Ion Batteries based on non-equilibrium thermodynamics

Keywords: lithium-ion battery, battery modeling, electrochemical simulation, concentrated electrolyte, ion transpor

(8 pages, 2010)

191. P. Popov, Y. Vutov, S. Margenov, O. Iliev Finite volume discretization of equations describing nonlinear diffusion in Li-Ion batteries

Keywords: nonlinear diffusion, finite volume discretization, Newton method, Li-lon batteries

(9 pages, 2010)

192. W. Arne, N. Marheineke, R. Wegener Asymptotic transition from Cosserat rod to string models for curved viscous inertial jets

Keywords: rotational spinning processes; inertial and viscous-inertial fiber regimes; asymptotic limits; slenderbody theory; boundary value problems

(23 pages, 2010)

193. L. Engelhardt, M. Burger, G. Bitsch Real-time simulation of multibody-systems for on-board applications

Keywords: multibody system simulation, real-time simulation, on-board simulation, Rosenbrock methods (10 pages, 2010)

\section{M. Burger, M. Speckert, K. Dreßler} Optimal control methods for the calculation of invariant excitation signals for multibody systems

Keywords: optimal control, optimization, mbs simulation, invariant excitation

(9 pages, 2010)
195. A Latz, J. Zausch

Thermodynamic consistent transport theory of Li-lon batteries

Keywords: Li-lon batteries, nonequilibrium thermodynamics, thermal transport, modeling

(18 pages, 2010)

\section{S. Desmettre}

Optimal investment for executive stockholders with exponential utility Keywords: portfolio choice, executive stockholder work effort, exponential utility

(24 pages, 2010)

197. W. Arne, N. Marheineke, J. Schnebele, R. Wegener

Fluid-fiber-interactions in rotational spinning process of glass wool production Keywords: Rotational spinning process, viscous thermal jets, fluid-fiber-interactions, two-way coupling, slenderbody theory, Cosserat rods, drag models, boundary value problem, continuation method

(20 pages, 2010)

198. A. Klar, J. Maringer, R. Wegener

A 3d model for fiber lay-down in nonwoven production processes

Keywords: fiber dynamics, Fokker-Planck equations, diffusion limits

(15 pages, 2010)

199. Ch. Erlwein, M. Müller

A regime-switching regression model for hedge funds

Keywords: switching regression model, Hedge funds, optimal parameter estimation, filtering

(26 pages, 2011)

200. M. Dalheimer

Power to the people - Das Stromnetz der Zukunft

Keywords: Smart Grid, Stromnetz, Erneuerbare Energien, Demand-Side Management

(27 pages, 2011)

201. D. Stahl, J. Hauth

PF-MPC: Particle Filter-Model Predictive Control

Keywords: Model Predictive Control, Particle Filter, CSTR, Inverted Pendulum, Nonlinear Systems, Sequential Monte Carlo

(40 pages, 2011)

\section{G. Dimitroff, J. de Kock}

Calibrating and completing the volatility cube in the SABR Model

Keywords: stochastic volatility, SABR, volatility cube, swaption

(12 pages, 2011)

203. J.-P. Kreiss, T. Zangmeister

Quantification of the effectiveness of a safety function in passenger vehicles on the basis of real-world accident data Keywords: logistic regression, safety function, realworld accident data, statistical modeling (23 pages, 2011)

204. P. Ruckdeschel, T. Sayer, A. Szimayer Pricing American options in the Heston model: a close look on incorporating correlation

Keywords: Heston model, American options, moment matching, correlation, tree method

(30 pages, 2011) 
205. H. Ackermann, H. Ewe, K.-H. Küfer,

M. Schröder

Modeling profit sharing in combinatorial exchanges by network flows

Keywords: Algorithmic game theory, profit sharing, combinatorial exchange, network flows, budget balance, core

(17 pages, 2011)

Status quo: April 2011 